\title{
Probing the top Yukawa coupling at the LHC via associated production of single top and Higgs
}

\author{
Vernon Barger, ${ }^{a}$ Kaoru Hagiwara ${ }^{b, c}$ and Ya-Juan Zheng ${ }^{d}$ \\ ${ }^{a}$ Department of Physics, University of Wisconsin, \\ Madison, WI 53706, U.S.A. \\ ${ }^{b}$ Tsung-Dao Lee Institute, Shanghai Jiao Tong University, \\ Shanghai 200240, China \\ ${ }^{c}$ KEK Theory Center and Sokendai, \\ Tsukuba, Ibaraki 305-0801, Japan \\ ${ }^{d}$ Department of Physics and Astronomy, University of Kansas, \\ Lawrence, KS 66045, U.S.A. \\ E-mail: barger@pheno.wisc.edu, kaoru.hagiwara@kek.jp, yjzheng@ku.edu
}

ABSTRACT: We study Higgs boson production associated with single top or anti-top via $t$-channel weak boson exchange at the LHC. The process is an ideal probe of the top quark Yukawa coupling, because we can measure the relative phase of $h t t$ and $h W W$ couplings, thanks to the significant interference between the two amplitudes. By choosing the emitted $W$ momentum along the polar axis in the $t h(\bar{t} h)$ rest frame, we obtain the helicity amplitudes for all the contributing subprocesses analytically, with possible CP phase of the Yukawa coupling. We study the azimuthal asymmetry between the $W$ emission and the $W b(\bar{b}) \rightarrow t(\bar{t}) h$ scattering planes, as well as several $t$ and $\bar{t}$ polarization asymmetries as a signal of $\mathrm{CP}$ violating phase in the $h t t$ coupling. Both the azimuthal asymmetry and the polarization perpendicular to the scattering plane are found to have the opposite sign between the top and anti-top events. We identify the origin of the sign of asymmetries, and propose the possibility of direct $\mathrm{CP}$ violation test in $p p$ collisions by comparing the top and anti-top polarization at the LHC.

Keywords: Beyond Standard Model, CP violation, Heavy Quark Physics, Higgs Physics ARXIV EPRINT: 1912.11795 


\section{Contents}

1 Introduction 1

2 Helicity amplitudes $\quad 4$

3 Differential cross sections $\quad 12$

3.1 Selecting the $b$ and $\bar{b}$ momentum direction $\quad 13$

$\begin{array}{ll}3.2 \mathrm{Q} \text { and W distributions } & 15\end{array}$

$\begin{array}{lll}4 & \text { Azimuthal angle asymmety } & 17\end{array}$

5 Polarization asymmetries $\quad 22$

$6 \quad$ T-odd v.s. CPV asymmetry $\quad 27$

$\begin{array}{lll}7 & \text { Summary and discussion } & 32\end{array}$

$\begin{array}{ll}\text { A Helicity amplitudes and the top spin orientation } & 34\end{array}$

B Polarized $t$ and $\bar{t}$ decay distributions $\quad 35$

$\begin{array}{ll}\text { C Phase space } & 38\end{array}$

\section{Introduction}

The top quark Yukawa coupling of the $125 \mathrm{GeV}$ Higgs boson $(h)$ is the largest of the Standard Model (SM) couplings, and the precise measurement of its magnitude and properties is the important target of the LHC experiments. Measurements of the loop-induced $h g g$ and $h \gamma \gamma$ transitions constrain the top Yukawa, or $h t t$ coupling indirectly, if only the SM particles contribute to the vertices with the SM couplings. The observation of the associated production of the Higgs boson and the top quark pair at the LHC [1, 2] determines the $h t t$ coupling directly, constraining its magnitude to be within about $10 \%$ of the SM prediction.

In this paper, we study the possibility of measuring a possible $\mathrm{CP}$ violating phase of the $h t t$ coupling in the Higgs boson production associated with single top or anti-top at the LHC. The cross section is dominated by the so-called $t$-channel $W$ exchange process, where the $W$ boson emitted from a quark or anti-quark in a proton scatters with a $b$ or $\bar{b}$ quark in the other proton to produce a pair of $h$ and $t$, or $\bar{t}$. The process is particularly sensitive to the phase of the $h t t$ coupling, because we can measure the real and imaginary part of the $h t t$ coupling through the interference between the amplitudes with the $h t t$ and $h W W$ couplings which have the same order of magnitude with opposite sign [3, 4] in the SM limit. 
We can therefore measure the phase of the $h t t$ coupling with respect to that of the $h W W$ coupling, whose magnitude and phase have already been constrained rather well [5-7] and will be determined precisely in the HL-LHC era at the level of $4 \%$ or better [8].

We adopt the following minimal non-SM modification to the top Yukawa coupling,

$$
\mathcal{L}_{h t t}=-g_{h t t} h \bar{t}\left(\cos \xi_{h t t}+i \sin \xi_{h t t} \gamma_{5}\right) t=-g_{h t t} h\left\{e^{-i \xi_{h t t}} t_{R}^{\dagger} t_{L}+e^{i \xi_{h t t}} t_{L}^{\dagger} t_{R}\right\},
$$

where we introduce the positive $\kappa$ factor as

$$
g_{h t t}=\left(m_{t} / v\right) \kappa_{h t t}>0
$$

for the normalization of the coupling. The Lagrangian expressed in terms of the chiral two-spinors $t_{L}$ and $t_{R}$

$$
\frac{1-\gamma_{5}}{2} t=\left(\begin{array}{c}
t_{L} \\
0
\end{array}\right), \quad \frac{1+\gamma_{5}}{2} t=\left(\begin{array}{c}
0 \\
t_{R}
\end{array}\right),
$$

show that $\xi_{h t t}$ is the CP phase of the Yukawa interactions. Its defined range is

$$
-\pi<\xi_{h t t} \lesssim \pi
$$

with respect to the $h W W$ coupling term

$$
\mathcal{L}_{h W W}=g_{h W W} h W_{\mu}^{-} W^{+\mu}
$$

for which we take the real positive value

$$
g_{h W W}=\left(2 m_{W}^{2} / v\right) \kappa_{h W W}>0 .
$$

$\mathrm{CP}$ violation in the $h t t$ coupling, $\xi_{h t t} \neq 0$, with $\kappa_{h t t} \neq 1$ can arise by radiative effects in the $h t t$ vertex due to new interactions which violate $\mathrm{CP}$, or in models with two or more Higgs doublets when the Higgs interactions violate CP. Once the underlying new physics model is fixed, we often obtain correlations among the non-SM effective couplings, such as $\kappa_{h W W}, \kappa_{h t t}, \xi_{h t t}$, and also for the other $h f f$ couplings as well as the loop induced $h g g, h \gamma \gamma$ and $h Z \gamma$ vertices. In this report, we set

$$
\kappa_{h t t}=\kappa_{h W W}=1
$$

in all the numerical results, in order to focus on the observable $\mathrm{CP}$ violating effects for relatively small phase

$$
\left|\xi_{h t t}\right| \lesssim 0.1 \pi
$$

While we emphasize the detection of small CPV effects, we present the helicity amplitudes for arbitrary real values of the three parameters, $\kappa_{h W W}, \kappa_{h t t}$ and $\xi_{h t t}$.

In figure 1, we show the total cross section of the Higgs boson production with single $t$ or $\bar{t}$ via $t$-channel $W$ exchange in $p p$ collisions at $\sqrt{s}=13 \mathrm{TeV}$ for the effective $h t t$ coupling of eq. (1.1), with $\kappa_{h t t}=1$ and $\left|\xi_{h t t}\right|$ between 0 (SM) and $\pi$. Also shown is the total cross section for $h$ and a $t \bar{t}$ pair in the same model. They are obtained by MadGraph [9] with 


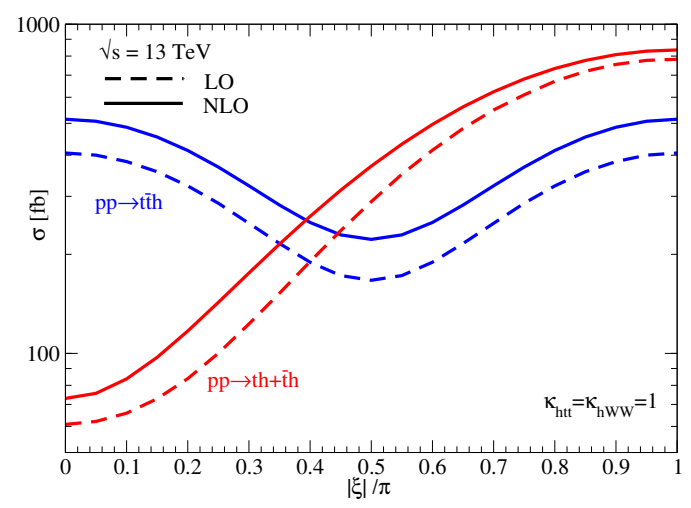

Figure 1. LO cross section at the LHC $(\sqrt{s}=13 \mathrm{TeV})$ for the sum of $p p \rightarrow t h$ and $p p \rightarrow \bar{t} h$ production via $t$-channel $W$ exchange (red curves) and for $p p \rightarrow t \bar{t} h$ production (blue curves) are shown as functions of the CP phase $\left|\xi_{h t t}\right|$ for $\kappa_{h t t}=1$. The LO (dashed curves) and the NLO (solid curves) predictions are obtained by Madgraph based on the study of ref. [12].

the effective Lagrangian of eq. (1.1) in Feynrules [10]. Here, and in all the following numerical calculations, we set $m_{h}=125 \mathrm{GeV}, m_{t}=173 \mathrm{GeV}, m_{W}=80.4 \mathrm{GeV}, v=246 \mathrm{GeV}$, $4 \pi / e^{2}=128$ and $\sin ^{2} \theta_{W}=0.233$ for the electroweak couplings. Factorization scale is set at $\mu=\left(m_{t}+m_{h}\right) / 4$ for the $h t$ and $h \bar{t}$ production via $t$-channel $W$ exchange processes in 5 -flavor QCD, following ref. [11]. As for the QCD production of $h t \bar{t}$ processes, we set the factorization and renormalization scales both at $\mu=\left(2 m_{t}+m_{h}\right) / 2$, following ref. [12]. The QCD coupling at $\mu=m_{Z}$ is set at $\alpha_{s}\left(m_{Z}\right)=0.118$ [13].

As is well known, the cross sections for the Higgs production with single $t$ or $\bar{t}$ are sensitive to the relative sign of the $h t t$ and the $h W W$ couplings, which becomes 13 times larger than the SM value at $\left|\xi_{h t t}\right|=\pi$ where the sign of the $h t t$ coupling is reversed [3]. Because of this huge enhancement factor, LHC experiments [14-18] have ruled out the region around $\left|\xi_{h t t}\right| \sim \pi$ for $\kappa_{h t t}=1$. It is worth noting, however, that we focus our attention in this paper on a relatively small magnitude of the CP phase $\left|\xi_{h t t}\right| \lesssim 0.1 \pi$, where the total cross sections do not deviate much from the SM values, $\sigma(t h+\bar{t} h)=60.85 \mathrm{fb}$ and $\sigma(t \bar{t} h)=406.26 \mathrm{fb}$ in the LO, as shown in figure 1 . Note also that the single $t$ or $\bar{t}+h$ production cross section is bigger than the $t \bar{t} h$ production cross section when $\left|\xi_{h t t}\right| \gtrsim 0.4 \pi$. Since only the $t \bar{t} h$ production has been observed, it is likely that the $\left|\xi_{h t t}\right| \lesssim 0.4 \pi$ region is preferred. Although we study the amplitudes in the small $\left|\xi_{h t t}\right| \lesssim 0.1 \pi$ region, it is straightforward to obtain all the asymmetry observables for an arbitrary $\xi_{h t t}$ by using our analytic amplitudes. Note, however, that the $\xi_{h t t}$-dependence of the asymmetries at large $\xi_{h t t}$ is determined by the real and imaginary parts of the amplitudes in the relevant region, which can be significantly different from the SM $\left(\xi_{h t t}=0\right)$ amplitudes studied carefully in this paper.

Past studies of the $h$ and single $t$ or $\bar{t}$ production signal and backgrounds at hadron colliders include NLO corrections with the matching between the 4- and 5-flavor QCD predictions [11, 19], with Higgs decay channels $h \rightarrow W W / Z Z[20,21], \gamma \gamma[21-24], b \bar{b}$ [21, 25-27] and $\tau^{+} \tau^{-}$[21]. CP phases of the top Yukawa couplings [28] are studied in $t+h$ production [21-24, 29, 30], $h t \bar{t}$ production [24], and in the loop induced vertices $h g g$ or $h \gamma \gamma$ [31]. The first result of our study has been reported in [32], and related studies are found in [33]. 
The paper is organized as follows. In section 2, we give helicity amplitudes for all the four LO subprocesses analytically. In section 3 , we study event distributions of $h t$ and $h \bar{t}$ production with a tagged forward jet, and show the exchanged $W$ helicity decomposition in $\mathbf{Q}$ (the virtual $W$ mass) and $\mathrm{W}$ (the invariant mass of the $t h$ or $\bar{t} h$ system) distributions. In section 4 , we study the azimuthal angle asymmetry between the $W$ emission plane and the $W^{+} b \rightarrow t h$ or $W^{-} \bar{b} \rightarrow \bar{t} h$ production plane about the $W$ momentum direction. In section 5 , we study $t$ and $\bar{t}$ polarizations in the $t(\bar{t})$ rest frames, as a function of $\mathbf{Q}, \mathbf{W}$ and the $W^{+} b \rightarrow t h\left(W^{-} \bar{b} \rightarrow \bar{t} h\right)$ scattering angle $\theta^{*}$ in the $t h(\bar{t} h)$ rest frame. In section 6 , we study consequences of $\mathrm{T}$ and $\mathrm{CP}$ transformations, and show the possibility that $\mathrm{CP}$ violation signal can be distinguished from T-odd asymmetry arising from the final state scattering phase in $p p$ collisions, by measuring the $t$ and $\bar{t}$ polarizations perpendicular to the scattering plane. The last section 7 gives a summary of our findings and remarks on possible measurements at HL-LHC. Appendix A gives the relation between the helicity amplitudes and $t$ and $\bar{t}$ spin polarizations, and appendix B gives polarized $t$ and $\bar{t}$ decay distributions.

\section{Helicity amplitudes}

In the SM, four subprocess contribute to single top plus Higgs production in the leading order

$$
\begin{array}{ll}
u b \rightarrow d t h & (c b \rightarrow s t h) \\
\bar{d} b \rightarrow \bar{u} t h & (\bar{s} b \rightarrow \bar{c} t h)
\end{array}
$$

and also to single anti-top plus Higgs production;

$$
\begin{array}{ll}
d \bar{b} \rightarrow u \bar{t} h & (s \bar{b} \rightarrow c \bar{t} h) \\
\bar{u} \bar{b} \rightarrow \bar{d} \bar{t} h & (\bar{c} \bar{b} \rightarrow \bar{s} \bar{t} h)
\end{array}
$$

We work in 5-flavor QCD with massless $b$-quark distribution in the proton, where the matching with the 4-flavor QCD with massive $b$-quark has been shown for the single $t$ plus $h$ processes in the NLO level $[12,20]$. The subprocesses in the parenthesis with second generation quarks have exactly the same matrix elements when we ignore quark mass and CKM mixing effects.

The Feynman diagrams of the subprocess $u b \rightarrow d t h$ in eq. (2.1a) are shown in figure 2. The left diagram ( $a$ ) has the $h W W$ coupling, while the right diagram $(b)$ has the $h t t$ coupling. The $u \rightarrow d W^{+}$emission part is common to both diagrams. The amplitudes for all the other subprocesses in eq. (2.1) are obtained by replacing the $u \rightarrow d W^{+}$emission current by $c \rightarrow s W^{+}, \bar{d} \rightarrow \bar{u} W^{+}$and $\bar{s} \rightarrow \bar{c} W^{+}$current, respectively. The Feynman diagrams for anti-top plus Higgs production in eq. (2.2) are obtained simply by replacing the $W^{+}$emission currents by the $W^{-}$emission currents, and by reversing the fermionnumber flow along the $b$ to $t$ transitions to make them $\bar{b}$ to $\bar{t}$ transitions. 

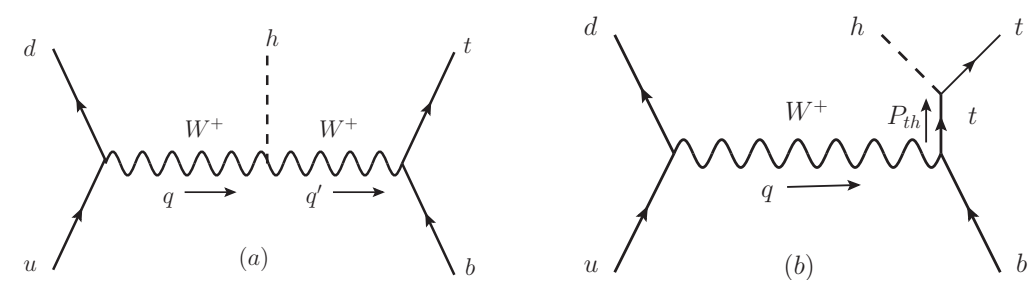

Figure 2. Feynman diagrams of $u b \rightarrow d t h$ subprocess. The four momenta $q^{\mu}$ and $q^{\prime \mu}$ along the $W^{+}$and $P_{t h}^{\mu}$ along the top propagators are shown with arrows.

In $p p$ collisions, valence quark initiated subprocesses $u b \rightarrow d t h$ (3a) and $d \bar{b} \rightarrow u \bar{t} h$ (4a) dominate the single top and anti-top production cross sections, respectively. The amplitudes for the subprocess $u b \rightarrow d t h$ in figure 2 are simply

$$
\mathcal{M}_{\sigma}=-\frac{g^{2}}{2} \bar{u}\left(p_{d}\right) \gamma_{\mu} \frac{1-\gamma_{5}}{2} u\left(p_{u}\right) D_{W}^{\mu \nu}(q) \bar{u}\left(p_{t}, \sigma\right) T_{\nu} \frac{1-\gamma_{5}}{2} u\left(p_{b}\right)
$$

with

$$
T^{\nu}=g_{h t t}\left(\cos \xi+i \sin \xi \gamma_{5}\right)\left(\not P_{t h}+m_{t}\right) D_{t}\left(P_{t h}\right) \gamma^{\nu}-g_{h W W} D_{W}^{\nu \rho}\left(q^{\prime}\right) \gamma_{\rho}
$$

for the effective top Yukawa coupling of eq. (1.1) and the SM $h W W$ coupling of eq. (1.6). The propagator factors

$$
D_{W}^{\mu \nu}(q)=\left(-g^{\mu \nu}+\frac{q^{\mu} q^{\nu}}{m_{W}^{2}}\right) D_{W}(q)
$$

and $D_{W}^{\nu \rho}\left(q^{\prime}\right)$ are the $W$-propagators, with $D_{W}(q)=\left(q^{2}-m_{W}^{2}\right)^{-1}$, and $D_{t}\left(P_{t h}\right)=\left(P_{t h}^{2}-m_{t}^{2}\right)^{-1}$ is for the top quark. The four momenta are depicted in figure 2 as

$$
q=p_{u}-p_{d}, \quad q^{\prime}=q-p_{h}=p_{t}-p_{b}, \quad P_{t h}=q+p_{b}=p_{t}+p_{h} .
$$

In the limit of neglecting all the quark masses except the top quark mass, $m_{t}$, the amplitudes depend only on the top quark helicity $\sigma / 2$ for $\sigma= \pm 1$, since only the left-handed quarks and right-handed anti-quarks contribute to the SM charged currents in the massless limit.

Because the $W^{+}$emission current of massless $u$ and $d$ quarks is conserved, only the spin 1 components of off-shell $W^{+}$propagates in the common $D_{W}^{\mu \nu}(q)$ term in eq. (2.5):

$$
-g^{\mu \nu}+\frac{q^{\mu} q^{\nu}}{m_{W}^{2}} \rightarrow-g^{\mu \nu}+\frac{q^{\mu} q^{\nu}}{q^{2}}=\sum_{\lambda= \pm 1,0}(-1)^{\lambda+1} \epsilon^{\mu}(q, \lambda)^{*} \epsilon^{\nu}(q, \lambda),
$$

where $\lambda$ denotes the helicity of virtual $W^{+}$, and the $(-1)^{\lambda+1}$ factor appears for $q^{2}<0$. By replacing the covariant propagation factor in the common $W^{+}$propagator with eq. (2.7), we can express the amplitudes eq. (2.3) as a sum over the contributions of the three $W^{+}$ helicity states:

$$
\mathcal{M}_{\sigma}=\sum_{\lambda= \pm 1,0} \mathcal{J}_{\lambda}\left(u \rightarrow d W_{\lambda}^{+}\right) \mathcal{T}_{\lambda \sigma}\left(W_{\lambda}^{+} b \rightarrow t_{\sigma} h\right)
$$

with

$$
\mathcal{J}_{\lambda}=\frac{g}{\sqrt{2}} D_{W}(q) \bar{u}\left(p_{d}\right) \gamma^{\mu} \frac{1-\gamma_{5}}{2} u\left(p_{u}\right) \epsilon_{\mu}^{*}(q, \lambda)(-1)^{\lambda}
$$




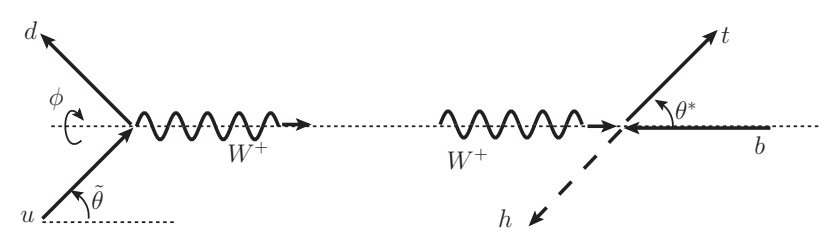

Figure 3. Scattering angles $\tilde{\theta}, \phi$ and $\theta^{*}$. The polar angle $\tilde{\theta}$ is defined in the Breit frame, whereas $\theta^{*}$ is defined in the $W^{+} b$ rest frame, for the common polar axis along the $W$ momentum direction. The azimuthal angle $\phi$ is the angle between the emission plane and the scattering plane.

and

$$
\mathcal{T}_{\lambda \sigma}=\frac{g}{\sqrt{2}} \bar{u}\left(p_{t}, \sigma\right)\left\{g_{h t t}\left(\cos \xi+i \sin \xi \gamma_{t}\right) D_{t}\left(P_{t h}\right)\left(P_{t h}+m_{t}\right) \gamma^{\nu}-g_{h W W} D_{W}^{\nu \rho}\left(q^{\prime}\right) \gamma_{\rho}\right\} \frac{1-\gamma_{5}}{2} u\left(p_{b}\right) \epsilon_{\nu}(q, \lambda)
$$

We calculate the helicity amplitudes $\mathcal{T}_{\lambda \sigma}$ for $W^{+} b \rightarrow t h$ process in the $W^{+} b$ or $t h$ rest frame, where the four momenta are parametrized as follows

$$
\begin{aligned}
q & =\frac{\mathrm{W}}{2}\left(1-\frac{\mathrm{Q}^{2}}{\mathrm{~W}^{2}}, 0,0,1+\frac{\mathrm{Q}^{2}}{\mathrm{~W}^{2}}\right)=\left(q^{0 *}, 0,0, q^{*}\right), \\
p_{b} & =\frac{\mathrm{W}}{2}\left(1+\frac{\mathrm{Q}^{2}}{\mathrm{~W}^{2}}, 0,0,-\left(1+\frac{\mathrm{Q}^{2}}{\mathrm{~W}^{2}}\right)\right)=\left(q^{*}, 0,0,-q^{*}\right), \\
p_{t} & =\frac{\mathrm{W}}{2}\left(1+\frac{m_{t}^{2}-m_{h}^{2}}{\mathrm{~W}^{2}}, \bar{\beta} \sin \theta^{*}, 0, \bar{\beta} \cos \theta^{*}\right)=\left(E_{t}^{*}, p^{*} \sin \theta^{*}, 0, p^{*} \cos \theta^{*}\right), \\
p_{h} & =\frac{\mathrm{W}}{2}\left(1+\frac{m_{h}^{2}-m_{t}^{2}}{\mathrm{~W}^{2}},-\bar{\beta} \sin \theta^{*}, 0,-\bar{\beta} \cos \theta^{*}\right)=\left(E_{h}^{*},-p^{*} \sin \theta^{*}, 0,-p^{*} \cos \theta^{*}\right) .
\end{aligned}
$$

Here, $\mathrm{W}=\sqrt{\left(p_{t}+p_{h}\right)^{2}}=\sqrt{P_{t h}^{2}}=m_{t h}$ is the invariant mass of the $t+h$ system, $\mathrm{Q}=\sqrt{-q^{2}}$ is the invariant mass of the virtual $W^{+}$, and $p^{*}=(\mathrm{W} / 2) \bar{\beta}$ is the common c.m. momentum of $t$ and $h$. The scattering angle $\theta^{*}$ of the top quark is measured from the $W^{+}$momentum direction as shown in figure 3. Because the top quark helicity amplitudes are calculated in this frame, all the polarization asymmetries presented in this paper should be confronted against data in the th rest frame of figure 3. On the other hand, because massless quark helicities are Lorentz invariant, and the $W^{+}$helicity is boost invariant along the $W^{+}$ momentum direction, which we take as the polar axis in figure 3 , we can evaluate the $u \rightarrow d W^{+}$emission amplitudes in the Breit frame [34], where the $W^{+}$four momentum has only the helicity axis component

$$
q^{\mu}=(0,0,0, \mathbf{Q})
$$

The $u$ and $d$ quark four momenta are

$$
\begin{aligned}
p_{u}^{\mu} & =\tilde{\omega}(1, \sin \tilde{\theta} \cos \phi,-\sin \tilde{\theta} \sin \phi, \cos \tilde{\theta}), \\
p_{d}^{\mu} & =\tilde{\omega}(1, \sin \tilde{\theta} \cos \phi,-\sin \tilde{\theta} \sin \phi,-\cos \tilde{\theta}),
\end{aligned}
$$


where their common energy $\tilde{\omega}$ and the reflecting momentum along the polar axis are, respectively,

$$
\begin{aligned}
\tilde{\omega} & =(\mathrm{Q} / 2)\left[2 \hat{s} /\left(\mathrm{W}^{2}+\mathrm{Q}^{2}\right)-1\right], \\
\tilde{\omega} \cos \tilde{\theta} & =\mathrm{Q} / 2,
\end{aligned}
$$

with $\hat{s}=\left(p_{u}+p_{b}\right)^{2}$ and $\mathrm{W}=m_{t h}$. In eq. (2.13) and in figure 3 , the $u \rightarrow d W^{+}$emission plane is rotated by $-\phi$ about the $z$-axis, so that the top quark azimuthal angle measured from the $u \rightarrow d W^{+}$emission plane is $\phi$.

The $u \rightarrow d W^{+}$emission amplitudes have very compact and intuitive expressions in the Breit frame:

$$
\begin{aligned}
& \mathcal{J}_{ \pm}=\frac{g}{\sqrt{2}} D_{W}(q)(2 \tilde{\omega})\left(e^{ \pm i \phi} \frac{1 \mp \cos \tilde{\theta}}{\sqrt{2}}\right) \\
& \mathcal{J}_{0}=\frac{g}{\sqrt{2}} D_{W}(q)(2 \tilde{\omega}) \sin \tilde{\theta}
\end{aligned}
$$

Here we adopt

$$
\epsilon^{\mu}(q, \lambda= \pm 1)=\frac{1}{\sqrt{2}}(0,1, \pm i, 0), \quad \epsilon^{\mu}(q, \lambda=0)=(1,0,0,0),
$$

for the three polarization vectors, which differs by the sign of the $\lambda=+1$ vector from the standard Jacob-Wick convention. The convention dependence cancels in the product, and our choice makes CP transformation properties of the sub-amplitudes, $\mathcal{J}_{\lambda}$ and $\mathcal{T}_{\lambda \sigma}$, simple because

$$
\epsilon^{\mu}(q, \lambda)^{*}=\epsilon^{\mu}(q,-\lambda) .
$$

It is interesting to note [34] that the $u \rightarrow d W^{+}$emission amplitudes can be expressed in terms of Wigner's $d$-functions. In terms of the invariants, they are expressed as

$$
\begin{aligned}
(2 \tilde{\omega}) \frac{1+\cos \tilde{\theta}}{2} & =\frac{\mathrm{Q}}{1-x+\mathrm{Q}^{2} / \hat{s}} \\
(2 \tilde{\omega}) \frac{\sin \tilde{\theta}}{\sqrt{2}} & =\frac{\mathrm{Q}}{1-x+\mathrm{Q}^{2} / \hat{s}} \sqrt{x-\mathrm{Q}^{2} / \hat{s}} \\
(2 \tilde{\omega})(1-\cos \tilde{\theta}) & =\frac{\mathrm{Q}}{1-x+\mathrm{Q}^{2} / \hat{s}}\left(x-\mathrm{Q}^{2} / \hat{s}\right)
\end{aligned}
$$

where

$$
x=1-\mathrm{W}^{2} / \hat{s}
$$

is the energy fraction of the $d$-quark in the $u b$ collision rest frame. It should be noted that for typical events with $x \lesssim 0.1$, the ordering

$$
\frac{1+\cos \tilde{\theta}}{2} \gg \frac{\sin \tilde{\theta}}{\sqrt{2}} \gg \frac{1-\cos \tilde{\theta}}{2}
$$

holds among the magnitudes of the $d$-functions. In particular, $J_{-}$for the $W^{+}$with helicity $\lambda=-1$ dominates over $J_{+}$for $\lambda=+1$, because a left-handed quark tends to emit a left-handed $W$ boson in the forward direction. 
The helicity amplitudes $\mathcal{T}_{\lambda \sigma}$ for $W_{\lambda}^{+} b \rightarrow t_{\sigma} h$ process are calculated in the $t h$ rest frame. We first express $\mathcal{T}_{\lambda \sigma}$ (2.10) in terms of chiral two-spinors [35]

$$
\begin{aligned}
\mathcal{T}_{\lambda \sigma}= & \frac{g}{\sqrt{2}} g_{h t t} D_{t}\left(P_{t h}\right)\left[e^{-i \xi} u_{R}^{\dagger}\left(p_{t}, \sigma\right) P \cdot \sigma_{+}+e^{i \xi} m_{t} u_{L}^{\dagger}\left(p_{t}, \sigma\right)\right] \epsilon(q, \lambda) \cdot \sigma_{-} u_{L}\left(p_{b}\right) \\
& +\frac{g}{\sqrt{2}} g_{h W W} D_{W}\left(q^{\prime}\right)\left[u_{L}^{\dagger}\left(p_{t}, \sigma\right) \epsilon(q, \lambda) \cdot \sigma_{-}+\frac{m_{t}}{m_{W}^{2}} p_{b} \cdot \epsilon(q, \lambda) u_{R}^{\dagger}\left(p_{t}, \sigma\right)\right] u_{L}\left(p_{b}\right)
\end{aligned}
$$

where we denote $P=P_{t h}=p_{t}+p_{h}, \xi=\xi_{h t t}$, and $\sigma_{ \pm}^{\mu}=(1, \pm \vec{\sigma})$ are the chiral four-vectors of $\sigma$ matrices. The upper term in eq. (2.21) is proportional to the Yukawa coupling, which flips the left-handed chirality of the incoming $b$-quark. Therefore, the first term in the parenthesis, namely the right-handed top production with $e^{-i \xi}$ phase factor grows with $P$, while the second term, namely the left-handed top production with $e^{i \xi}$ factor is proportional to $m_{t}$ giving the extra chirality flip. As for the $W$-exchange amplitudes, the lower term in eq. (2.21) the chirality flip right-handed top production is non-negligible because of the scalar component of the exchanged $W$ boson, which has the $1 / m_{W}^{2}$ factor and non-conservation of the $t b$ current, whose divergence gives $m_{t}$.

The amplitudes $\mathcal{T}_{\lambda \sigma}$ can be calculated straightforwardly in the thest frame of eq. (2.11), see figure 3 , giving

$$
\begin{aligned}
\mathcal{T}_{+ \pm}= & \pm \frac{g}{\sqrt{2}} g_{h W W} D_{W}\left(q^{\prime}\right) \frac{m p^{*}}{m_{W}^{2}} \sqrt{q^{*}\left(E^{*} \pm p^{*}\right)\left(1 \pm \cos \theta^{*}\right)} \frac{\sin \theta^{*}}{\sqrt{2}} \\
\mathcal{T}_{- \pm}= & \frac{g}{\sqrt{2}} g_{h W W} D_{W}\left(q^{\prime}\right)\left[ \pm \frac{m p^{*}}{m_{W}^{2}} \sqrt{q^{*}\left(E^{*} \pm p^{*}\right)\left(1 \pm \cos \theta^{*}\right)} \frac{\sin \theta^{*}}{\sqrt{2}}+\sqrt{2 q^{*}\left(E^{*} \mp p^{*}\right)\left(1 \mp \cos \theta^{*}\right)}\right] \\
& +\frac{g}{\sqrt{2}} g_{h t t} D_{t}(P)\left[\left(e^{-i \xi} \mathrm{W} \sqrt{E^{*} \pm p^{*}}+e^{i \xi} m \sqrt{E^{*} \mp p^{*}}\right) \sqrt{2 q^{*}\left(1 \mp \cos \theta^{*}\right)}\right] \\
\mathcal{T}_{0 \pm}= & \pm \frac{g}{\sqrt{2}} g_{h W W} D_{W}\left(q^{\prime}\right)\left[\left(\frac{m\left(q^{*} E_{h}^{*}+q^{0 *} p^{*} \cos \theta^{*}\right)}{m_{W}^{2} \mathrm{Q}} \sqrt{E^{*} \pm p^{*}}+\frac{\mathrm{W}}{\mathrm{Q}} \sqrt{E^{*} \mp p^{*}}\right) \sqrt{q^{*}\left(1 \pm \cos \theta^{*}\right)}\right] \\
& \pm \frac{g}{\sqrt{2}} g_{h t t} D_{t}(P) \frac{\mathrm{W}}{\mathrm{Q}}\left[\left(e^{-i \xi} \mathrm{W} \sqrt{E^{*} \pm p^{*}}+e^{i \xi} m \sqrt{E^{*} \mp p^{*}}\right) \sqrt{q^{*}\left(1 \mp \cos \theta^{*}\right)}\right]
\end{aligned}
$$

where we denote $m=m_{t}$ and $E^{*}=E_{t}^{*}$. Note that the term $\sqrt{E^{*}+p^{*}}$ appears when the top helicity matches its chirality, while $\sqrt{E^{*}-p^{*}}$ when they mismatch. The amplitude for $\lambda=+1$ does not have the top Yukawa coupling contribution because the angular momentum along the $z$-axis is $J_{z}=+\frac{3}{2}$ for the left-handed $b$-quark, which cannot couple to $J=1 / 2$ top quark. For $\lambda=-1$ and $\lambda=0 W^{+}$, both $W$ and $t$ exchange amplitudes contribute. Most importantly, the $\lambda=0$ amplitudes are enhanced by the factor of $\mathrm{W} / \mathrm{Q}$, which is a consequence of the boost factor of the longitudinally polarized $W^{+}$wave function with helicity $\lambda=0$. The polarization vectors in eq. (2.16) in the Breit frame are invariant for $\lambda= \pm 1$, but the longitudinal vector becomes

$$
\epsilon^{\mu}(q, \lambda=0)=\frac{1}{\mathbf{Q}}\left(q^{*}, 0,0, q^{0 *}\right)
$$

in the $t h$ rest frame, where both $q^{*}$ and $q^{0 *}$ are the order of $\mathrm{W} / 2$ as in eq. (2.11a). 
Summing over the three $W$ polarization contributions, we find the amplitudes [32]

$$
\begin{aligned}
\mathcal{M}_{+}= & \frac{1-\tilde{c}}{2} e^{i \phi} \sin \frac{\theta^{*}}{2}\left[\frac{1+\cos \theta^{*}}{4} \bar{\beta} A\right] \\
& +\frac{1+\tilde{c}}{2} e^{-i \phi} \sin \frac{\theta^{*}}{2}\left[\left(\frac{1+\cos \theta^{*}}{4} \bar{\beta}+\epsilon \delta \delta^{\prime}\right) A-\left(e^{-i \xi}+\delta \delta^{\prime} e^{i \xi}\right) B\right] \\
& \left.+\frac{\tilde{s} \mathrm{~W}}{2} \frac{\theta^{*}}{\mathrm{Q}} \cos \frac{\theta^{*}}{2}\left[\frac{q_{h}^{*}+q^{0 *} p^{*} \cos \theta^{*}}{\mathrm{~W}^{2}}+\epsilon \delta \delta^{\prime}\right) A-\left(e^{-i \xi}+\delta \delta^{\prime} e^{i \xi}\right) B\right], \\
\mathcal{M}_{-}= & -\frac{1-\tilde{c}}{2} e^{i \phi} \cos \frac{\theta^{*}}{2}\left[\frac{1-\cos \theta^{*}}{4} \bar{\beta} A\right] \delta \\
& -\frac{1+\tilde{c}}{2} e^{-i \phi} \cos \frac{\theta^{*}}{2}\left[\left(\frac{1-\cos \theta^{*}}{4} \bar{\beta}-\epsilon \frac{\delta^{\prime}}{\delta}\right) A+\left(e^{-i \xi}+\frac{\delta^{\prime}}{\delta} e^{i \xi}\right) B\right] \delta \\
& -\frac{\tilde{s} \mathrm{~W}}{2} \sin \frac{\theta^{*}}{2}\left[\left(\frac{q^{*} E_{h}^{*}+q^{0 *} p^{*} \cos \theta^{*}}{\mathrm{~W}^{2}}+\epsilon \frac{\delta^{\prime}}{\delta}\right) A-\left(e^{-i \xi}+\frac{\delta^{\prime}}{\delta} e^{i \xi}\right) B\right] \delta .
\end{aligned}
$$

where the factors

$$
\begin{aligned}
& A=2 g^{2} g_{h W W} \frac{m m_{t h}}{m_{W}^{2}} D_{W}(q) D_{W}\left(q^{\prime}\right) \tilde{\omega} \sqrt{2 q^{*}\left(E^{*}+p^{*}\right)}, \\
& B=-2 g^{2} g_{h t t} m_{t h} D_{W}(q) D_{t}(P) \tilde{\omega} \sqrt{2 q^{*}\left(E^{*}+p^{*}\right)}
\end{aligned}
$$

are chosen such that they are positive definite. The $\epsilon, \delta$, and $\delta^{\prime}$ factors are

$$
\epsilon=\frac{m_{W}^{2}}{m^{2}}, \quad \delta=\frac{m}{E^{*}+p^{*}}, \quad \delta^{\prime}=\frac{m}{\mathrm{~W}},
$$

where $\epsilon \simeq 0.21, \delta$ and $\delta^{\prime}$ are all small at large $\mathrm{W}$, and in particular, $\delta \simeq \delta^{\prime}$ holds rather accurately except in the vicinity of $t h$ production threshold, $\mathrm{W} \simeq m_{t}+m_{h}$. At $\mathrm{W} \gtrsim 400 \mathrm{GeV}$, the amplitudes are well approximated as

$$
\begin{aligned}
\mathcal{M}_{+} \sim & {\left[\frac{1+\tilde{c}}{2} e^{-i \phi} \sin \frac{\theta^{*}}{2}+\frac{\mathrm{W}}{\mathrm{Q}} \frac{\tilde{s}}{2} \cos \frac{\theta^{*}}{2}\right]\left[\frac{1+\cos \theta^{*}}{4} A-e^{-i \xi} B\right], } \\
\mathcal{M}_{-} \sim & -\frac{1+\tilde{c}}{2} \cos \frac{\theta^{*}}{2} e^{-i \phi}\left[\left(\frac{1-\cos \theta^{*}}{4}-\epsilon\right) A+2 \cos \xi B\right] \delta \\
& -\frac{\mathrm{W} \tilde{s}}{\mathrm{Q}} \frac{\sin }{2} \sin \frac{\theta^{*}}{2}\left[\left(\frac{1+\cos \theta^{*}}{4}+\epsilon\right) A-2 \cos \xi B\right] \delta,
\end{aligned}
$$

where we have dropped $\lambda=+1$ contributions which are suppressed at high $\mathrm{W} / \mathrm{Q}$. The above approximations show that the leading $\lambda=0$ contributions with the $\mathrm{W} / \mathrm{Q}$ enhancement factor are proportional to

$$
\begin{array}{ll}
\frac{1+\cos \theta^{*}}{4} A-e^{-i \xi} B & \text { for } \mathcal{M}_{+} \\
\left(\frac{1+\cos \theta^{*}}{4}+\epsilon\right) A-2 \cos \xi B & \text { for } \mathcal{M}_{-} .
\end{array}
$$


Because both $A$ and $B$ terms are positive definite, their magnitudes are smallest at $\xi=0$ (SM), where the $W$ exchange term $A$ and the $t$-exchange term $B$ interfere destructively, whereas they become largest at $|\xi|=\pi$ where the two terms interfere constructively, explaining the order of magnitude difference in the total cross section between $\xi=0$ and $|\xi|=\pi$ shown in figure 1 . This strong interference between the two amplitudes gives the opportunity to accurately measure the $h t t$ Yukawa coupling with respect to the $h W W$ coupling.

Another important observation from the above approximation is that the $\mathrm{CP}$-violation (CPV) effects proportional to $\sin \xi$ are significant only in the amplitude of the right-handed helicity top quark, $\mathcal{M}_{+}$, because $\mathcal{M}_{-}$is proportional to $e^{-i \xi}+e^{+i \xi}=2 \cos \xi$ at large $\mathrm{W}$ where $\delta=\delta^{\prime}$. We note here that $\mathcal{M}_{+}$is the leading helicity amplitude at large $\mathrm{W}$, where the chirality flip Yukawa interactions give right-handed top quark from the left-handed $b$-quark. The negative helicity amplitudes $\mathcal{M}_{-}$is suppressed by an additional chirality flip of the top quark, indicated by the factor $\delta=m /$ w in eq. (2.27b).

Before starting discussions about signals at the LHC, let us complete all the helicity amplitudes of the contributing subprocess for both th and $\bar{t} h$ productions. First, the amplitudes for the subprocesses $c b \rightarrow$ sth are the same as those for $u b \rightarrow d t h$ in our approximation of neglecting quark masses and CKM mixing:

$$
\mathcal{M}_{\sigma}(u b \rightarrow d t h)=\mathcal{M}_{\sigma}(c b \rightarrow s t h)=\sum_{\lambda} \mathcal{J}_{\lambda} \mathcal{T}_{\lambda \sigma}
$$

as summarized in eqs. (2.24). There are two additional contributions to th production from the anti-quark distributions of proton

$$
\mathcal{M}_{\sigma}(\bar{d} b \rightarrow \bar{u} t h)=\mathcal{M}_{\sigma}(\bar{s} b \rightarrow \bar{c} t h)=\sum_{\lambda} \overline{\mathcal{J}}_{\lambda} \mathcal{T}_{\lambda \sigma}
$$

where the $\bar{d} \rightarrow \bar{u} W^{+}$emission amplitudes are

$$
\overline{\mathcal{J}}_{\lambda}=\frac{g}{\sqrt{2}} \bar{v}\left(p_{\bar{d}}\right) \gamma^{\mu} \frac{1-\gamma_{5}}{2} v\left(p_{\bar{u}}\right) \epsilon_{\mu}^{*}(q, \lambda)(-1)^{\lambda}
$$

In the Breit frame, they are expressed as

$$
\begin{aligned}
& \overline{\mathcal{J}}_{ \pm}=\frac{g}{\sqrt{2}} D_{W}(q)(2 \tilde{\omega})\left(e^{ \pm i \phi} \frac{1 \pm \cos \tilde{\theta}}{\sqrt{2}}\right) \\
& \overline{\mathcal{J}}_{0}=\frac{g}{\sqrt{2}} D_{W}(q)(2 \tilde{\omega}) \sin \tilde{\theta}
\end{aligned}
$$

We note the relation

$$
\overline{\mathcal{J}}_{\lambda}(\tilde{\theta}, \phi)=\mathcal{J}_{-\lambda}(\tilde{\theta},-\phi)=\mathcal{J}_{-\lambda}^{*}(\tilde{\theta}, \phi)
$$

between $\mathcal{J}_{\lambda}$ and $\overline{\mathcal{J}}_{\lambda}$. The matrix elements for the $W^{+}$emission from anti-quarks differ from those from quarks by simply replacing $1 \pm \tilde{c}$ by $1 \mp \tilde{c}$, thereby changing the preferred helicity of $W^{+}$from $\lambda=-1$ (for $u \rightarrow d W^{+}$and $c \rightarrow s W^{+}$) to $\lambda=+1$ (for $\bar{d} \rightarrow \bar{u} W^{+}$ and $\left.\bar{s} \rightarrow \bar{c} W^{+}\right)$. The $\lambda=0$ amplitude remains the same. Note that our special phase 
convention for the vector boson polarization vectors in eq. (2.16) allows the identities in eq. (2.33) to hold without the $\lambda$-dependent sign factor, $(-1)^{\lambda}$, that appears in the standard Jacob-Wick convention.

Now the $\bar{t} h$ production amplitudes are

$$
\begin{aligned}
& \overline{\mathcal{M}}_{\bar{\sigma}}(d \bar{b} \rightarrow u \bar{t} h)=\overline{\mathcal{M}}_{\bar{\sigma}}(s \bar{b} \rightarrow c \bar{t} h)=\sum_{\lambda} \mathcal{J}_{\lambda} \overline{\mathcal{T}}_{\lambda \bar{\sigma}} \\
& \overline{\mathcal{M}}_{\bar{\sigma}}(\bar{u} \bar{b} \rightarrow \bar{d} \bar{t} h)=\overline{\mathcal{M}}_{\bar{\sigma}}(\bar{c} \bar{b} \rightarrow \bar{s} \bar{t} h)=\sum_{\lambda} \overline{\mathcal{J}}_{\lambda} \overline{\mathcal{T}}_{\lambda \bar{\sigma}}
\end{aligned}
$$

where $\mathcal{J}_{\lambda}$ and $\overline{\mathcal{J}}_{\lambda}$ are the same as in eqs. (2.15) and (2.32), respectively, and the $W^{-} \bar{b} \rightarrow \bar{t} h$ amplitudes $\overline{\mathcal{T}}_{\lambda \bar{\sigma}}$ are obtained from $\mathcal{T}_{\lambda \sigma}$ by CP transformation

$$
\overline{\mathcal{T}}_{\lambda \bar{\sigma}}\left(\theta^{*}, \xi\right)=-\bar{\sigma} \mathcal{T}_{-\lambda,-\bar{\sigma}}\left(\theta^{*},-\xi\right)=-\bar{\sigma} \mathcal{T}_{-\lambda,-\bar{\sigma}}\left(\theta^{*}, \xi\right)^{*}
$$

Note that the first identity above tells the invariance of the amplitudes when all the initial and final states are CP transformed, along with the reversal of the sign of the CP phase. The latter equality is valid in our tree-level expressions eqs. (2.22) where absorptive parts of the amplitudes (including the top quark width) are set to be zero. ${ }^{1}$

It is instructive to compare the amplitudes of the two subprocesses which are related by CP transformation, such as between the amplitudes (2.24) or (2.29) for $u b \rightarrow d t h$ and those of eq. (2.34b) for $\bar{u} \bar{b} \rightarrow \bar{d} \bar{t} h$,

$$
\begin{aligned}
& \mathcal{M}_{\sigma}\left(u b \rightarrow d t h ; \tilde{\theta}, \phi, \theta^{*} ; \xi\right)=\sum_{\lambda} \mathcal{J}_{\lambda}(\tilde{\theta}, \phi) \mathcal{T}_{\lambda \sigma}\left(\theta^{*}, \xi\right) \\
& \overline{\mathcal{M}}_{\bar{\sigma}}\left(\bar{u} \bar{b} \rightarrow \bar{d} \bar{t} h ; \tilde{\theta}, \phi, \theta^{*} ; \xi\right)=\sum_{\lambda} \overline{\mathcal{J}}_{\lambda}(\tilde{\theta}, \phi) \overline{\mathcal{T}}_{\lambda \bar{\sigma}}\left(\theta^{*}, \xi\right)
\end{aligned}
$$

By using the identities (2.33) and (2.35) among the sub-amplitudes, we find the relation

$$
\overline{\mathcal{M}}_{-\sigma}\left(\bar{u} \bar{b} \rightarrow \bar{d} \bar{t} h ; \tilde{\theta}, \phi, \theta^{*} ; \xi\right)=\sigma \mathcal{M}_{\sigma}\left(u b \rightarrow d t h ; \tilde{\theta},-\phi, \theta^{*} ;-\xi\right)
$$

between $\overline{\mathcal{M}}_{\bar{\sigma}}$ and $\mathcal{M}_{\sigma}$, when $\bar{\sigma}=-\sigma$. It is worth noting that if we ignore the absorptive phase of the amplitudes, such as the top quark width in the propagator, the above identity gives

$$
\overline{\mathcal{M}}_{-\sigma}\left(\bar{u} \bar{b} \rightarrow \bar{d} \bar{t} h ; \tilde{\theta}, \phi, \theta^{*} ; \xi\right)=\sigma \mathcal{M}_{\sigma}\left(u b \rightarrow d t h ; \tilde{\theta}, \phi, \theta^{*} ; \xi\right)^{*},
$$

because both $\phi$ and $\xi$ appear in the amplitudes only as the phase factor, $e^{ \pm i \phi}$ and $e^{ \pm i \xi}$. This tells that all the distributions of the CP transformed processes are identical even in the presence of $\mathrm{CP}$-violating phase, $\xi \neq 0$, if we ignore the absorptive amplitudes from the

\footnotetext{
${ }^{1}$ Note that the sign factor, $-\bar{\sigma}$, in the identities (2.35) is a consequence of the phase convention of refs. $[35,36]$ where the $v$-spinors for anti-fermions are expressed as

$$
\begin{aligned}
& v_{L}(p, \bar{\sigma})=i \sigma^{2} u_{R}(p, \bar{\sigma})^{*}=-\bar{\sigma} \sqrt{E+\bar{\sigma} p} \chi_{-\bar{\sigma}}(\vec{p}) \\
& v_{R}(p, \bar{\sigma})=-i \sigma^{2} u_{L}(p, \bar{\sigma})^{*}=\bar{\sigma} \sqrt{E-\bar{\sigma} p} \chi_{-\bar{\sigma}}(\vec{p}) .
\end{aligned}
$$
}

See also appendix B of ref. [37]. 
final state interactions. We will discuss the origin of this somewhat unexpected property among the amplitudes in section 5 .

In $p p$ collisions, the dominant subprocess for single production of Higgs and antitop quark comes from the collision of valence down-quark scattering with $\bar{b}$ quark, whose amplitudes are given by eq. (2.34a). Since the properties of the $\bar{t} h$ production processes are governed by these amplitudes, we give their explicit form by using the same $A$ and $B$ factors of eq. (2.25):

$$
\begin{aligned}
\overline{\mathcal{M}}_{+}= & \frac{1-\tilde{c}}{2} e^{i \phi} \cos \frac{\theta^{*}}{2}\left[\left(\frac{1-\cos \theta^{*}}{4} \bar{\beta}-\epsilon \frac{\delta^{\prime}}{\delta}\right) A+\left(e^{i \xi}+\frac{\delta^{\prime}}{\delta} e^{-i \xi}\right) B\right] \delta \\
& +\frac{1+\tilde{c}}{2} e^{-i \phi} \cos \frac{\theta^{*}}{2} \frac{1-\cos \theta^{*}}{4} \bar{\beta} A \delta \\
& +\frac{\tilde{s} \mathrm{~W}}{2} \frac{\theta^{*}}{\operatorname{Q}} \sin \frac{\theta^{*}}{2}\left[\left(\frac{q^{*} E_{h}^{*}+q^{0 *} p^{*} \cos \theta^{*}}{\mathrm{~W}^{2}}+\epsilon \frac{\delta^{\prime}}{\delta}\right) A-\left(e^{i \xi}+\frac{\delta^{\prime}}{\delta} e^{-i \xi}\right) B\right] \delta \\
\overline{\mathcal{M}}_{-}= & \frac{1-\tilde{c}}{2} e^{i \phi} \sin \frac{\theta^{*}}{2}\left[\left(\frac{1+\cos \theta^{*}}{4} \bar{\beta}+\epsilon \delta \delta^{\prime}\right) A-\left(e^{i \xi}+\delta \delta^{\prime} e^{-i \xi}\right) B\right] \\
& +\frac{1+\tilde{c}}{2} e^{-i \phi} \sin \frac{\theta^{*}}{2} \frac{1+\cos \theta^{*}}{4} \bar{\beta} A \\
& \left.\left.+\frac{\tilde{s} \mathrm{~W}}{2} \frac{\theta^{*}}{\operatorname{Q}} \cos \frac{\theta^{*} E_{h}^{*}+q^{0 *} p^{*} \cos \theta^{*}}{2}+\epsilon \delta \delta^{\prime}\right) A-\left(e^{i \xi}+\delta \delta^{\prime} e^{-i \xi}\right) B\right]
\end{aligned}
$$

where the $d \rightarrow u W^{-}$emission amplitudes $\mathcal{J}_{\lambda}$ are the same as the $u \rightarrow d W^{+}$emission amplitudes in the $u b \rightarrow d t h$ subprocess amplitudes, eq. (2.24), while the $W^{-} \bar{b} \rightarrow \bar{t} h$ amplitudes $\overline{\mathcal{T}}_{\lambda \bar{\sigma}}$ are obtained from the $W^{+} b \rightarrow t h$ amplitudes $\mathcal{T}_{\lambda \sigma}$ by CP transformation in eq. (2.35). The chirality favored helicity of $\bar{t}$ from right-handed $\bar{b}$ is now $-1 / 2$, and the corresponding amplitude $\overline{\mathcal{M}}_{-}$has the leading $e^{i \xi}$ factor from the $t_{L}^{\dagger} t_{R}$ term in the Lagrangian eq. (1.1), while the contribution of the $e^{-i \xi_{t}} t_{R} t_{L}$ term is doubly chirality suppressed, by the $\delta \delta^{\prime}$ factor. In the helicity $+1 / 2$ amplitude $\overline{\mathcal{M}}_{+}$, single chirality flip (in addition to the flip due to the Yukawa interaction) is necessary, either in the spinor wave function (giving $\delta$ ), or in the top quark propagator (giving $\delta^{\prime}$ ). Summing up, we find $\overline{\mathcal{M}}_{-}$to have significant imaginary part proportional to $\sin \xi$, whereas $\overline{\mathcal{M}}_{+}$is almost proportional to $\cos \xi$, which are opposite of what we find for the single $t$ and $h$ production amplitudes.

\section{Differential cross sections}

The differential cross section in $p p$ collisions from the subprocess $u b \rightarrow d t h$ is given at leading order by

$$
d \sigma=\int d x_{1} \int d x_{2}\left[D_{u}\left(x_{1}, \mu\right) D_{b}\left(x_{2}, \mu\right)+D_{b}\left(x_{1}, \mu\right) D_{u}\left(x_{2}, \mu\right)\right] d \hat{\sigma}(u b \rightarrow d t h)
$$


where $D_{u}$ and $D_{b}$ are the PDF of the $u$ and $b$ quark, respectively, in the protons. The colliding parton momenta in LHC laboratory frame are

$$
\begin{aligned}
& p_{u}=\frac{\sqrt{s}}{2}\left(x_{1}, 0,0, x_{1}\right), \\
& p_{b}=\frac{\sqrt{s}}{2}\left(x_{2}, 0,0,-x_{2}\right),
\end{aligned}
$$

in the first term of eq. (3.1), whereas the $u$ - and $b$-quark four momenta are exchanged in the second term. Therefore, the sign of the $b$-quark momentum along the $z$-axis is negative for half of the events and positive for the other half. In order to perform the azimuthal angle or polarization asymmetry measurements proposed in [32], we should identify the momentum of the virtual $W^{+}$emitted from the $u$ (or $c, \bar{d}, \bar{s}$ ) quark. This is possible only when we can identify the sign of $p_{z}$ of the $b$-quark in the laboratory frame.

\subsection{Selecting the $b$ and $\bar{b}$ momentum direction}

Because the $u$ quark distribution is significantly harder than the $b$ quark distribution in the proton, when the sign of the $b$-quark momentum along the $z$-axis is negative, the rapidity of the colliding $u+b$ system $Y(u b)=Y(t h j)$ tends to be positive:

$$
Y(t h j)=\frac{1}{2} \ln \frac{E(t h j)+p_{z}(t h j)}{E(t h j)-p_{z}(t h j)}=\frac{1}{2} \ln \frac{x_{1}}{x_{2}} .
$$

Shown in figure 4(a) are the $Y$ distributions of the thj events where the light quark or anti-quark jet from the $t$-channel $W$ emission are tagged with cuts;

$$
p_{T}^{j}>30 \mathrm{GeV} \text {, and }\left|\eta_{j}\right|<4.5 \text {. }
$$

Events with negative $b$-quark $p_{z}$ are shown by solid curves, whereas those with positive $b$-quark $p_{z}$ are given by dashed curves. The dashed curves are obtained from the solid curves by reversing the sign of $Y(t h j)$, and hence the sum of all thj events should have symmetric $Y(t h j)$ distribution about $Y(t h j)=0$ as shown in solid black curve. The blue curves give the sum of $u b \rightarrow d t h$ and $c b \rightarrow s t h$ subprocess contributions (that have exactly the same matrix elements), and the red curves are for the sum of $\bar{d} b \rightarrow \bar{u} t h$ and $\bar{s} b \rightarrow \bar{c} t h$ subprocess contributions. As expected, events with $Y(t h j)>1$ are mostly from the $b$-quark with negative $p_{z}$ (blue and red solid curves). Although the purity (the probability) of negative $p_{z}$ of $b$-quark is $95 \%$, only $41 \%$ of the total events satisfy the $|Y(t h j)>1|$ cut, leaving $(59 \%)$ of the events with mixed kinematics which results in significant reduction of observable asymmetries and polarizations. The situation is much worse for $\bar{t} h j$ production processes, as shown in figure 4(b). With the same $|Y(t h j)>1|$ cut, the purity is only $89 \%$ and only $31 \%$ of the events are kept. It is mainly because the down quark is not as hard and populous as the up quark in the proton. All results in our study are calculated with the CTEQ14 PDF in the LO [38] with the factorization scale $\mu=\frac{m_{t}+m_{h}}{4}$, following ref. [11].

In figure 5(a) and 5(b), we show the tagged jet pseudo-rapidity distributions. Now the separation of events with negative $b$ momentum (shown by blue and red thick curves) and those with positive $b$ momentum (shown by blue and red thin curves) is clearer for both $t h j$ 

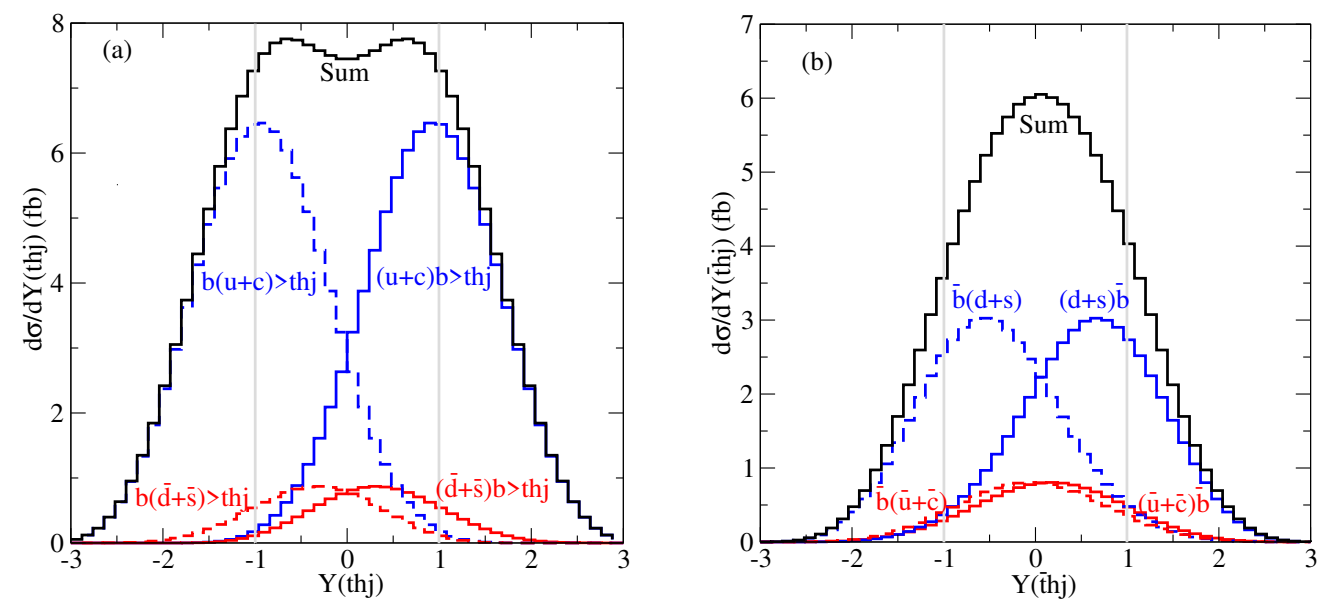

Figure 4. Rapidity distributions of the $t h j$ (a) and $\bar{t} h j$ (b) systems in $p p$ collisions at $\sqrt{s}=13 \mathrm{TeV}$. The blue curves are for contributions from subprocesses including the valence quark, whereas the red curves are for those from sea quark only. The events of $b(\bar{b})$ quark momentum direction along or opposite to the $z$ axis are shown by solid and dashed lines, respectively. The black curves are the sum of the rapidities from the four subprocesses. The quark and anti-quark jets from $t$-channel $\mathrm{W}$ emission are tagged with cuts $p_{T}^{j}>30 \mathrm{GeV}$, and $\left|\eta_{j}\right|<4.5$.
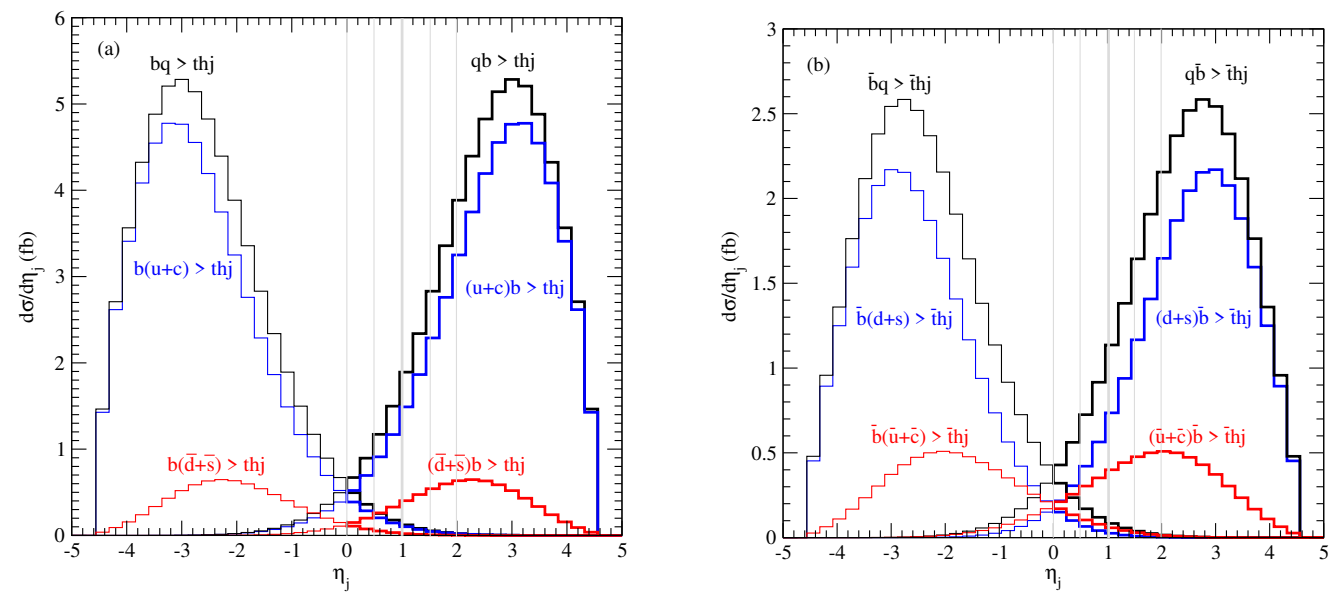

Figure 5. Tagged jet pseudo-rapidity distributions in $t h j$ (a) and $\bar{t} h j$ (b) events in $p p$ collisions at $\sqrt{s}=13 \mathrm{TeV}$.

(a) and $\bar{t} h j$ (b). In tables 1 and 2, we show the purity and the survival rate of several $\eta_{j}$ selection cuts for choosing events with negative $b$ or $\bar{b}$ momentum events, respectively, for $t h j$ and $\bar{t} h j$ processes. Even for $\eta_{j}>0$, when all events are used in the analysis, the purity is higher than $96 \%$ for both $t h j$ and $\bar{t} h j$ events. In this report, we adopt the selection cut

$$
1<\eta_{j}<4.5, \quad p_{T}^{j}>30 \mathrm{GeV}
$$

for the jet tag. Since the purity is higher than $99 \%$ for both thj (table 1) and $\bar{t} h j$ (table 2), we can safely neglect contribution from events with the wrong $b$-quark momentum direction, whose analysis requires additional kinematical considerations. We note here that 


\begin{tabular}{|c|c|c|c|c|c|c|c|c|c|c|}
\hline \multirow[t]{2}{*}{ Cut } & \multicolumn{3}{|c|}{$\sigma(q b \rightarrow t h j)[\mathrm{fb}]$} & \multicolumn{3}{|c|}{$\sigma(b q \rightarrow t h j)[\mathrm{fb}]$} & \multirow[t]{2}{*}{$\sigma(t h j)[\mathrm{fb}]$} & \multirow{2}{*}{$\begin{array}{c}\text { Purity }[\%] \\
q b\end{array}$} & \multicolumn{2}{|c|}{ Fraction in $q b[\%]$} \\
\hline & $u b+c b$ & $\bar{d} b+\bar{s} b$ & Sum & $b u+b c$ & $b \bar{d}+b \bar{s}$ & Sum & & & $u b+c b$ & $\bar{d} b+\bar{s} b$ \\
\hline$\eta_{j}>0$ & 12.74 & 1.75 & 14.49 & 0.32 & 0.076 & 0.40 & $14.89(100 \%)$ & 97.3 & 87.9 & 12.1 \\
\hline$\eta_{j}>0.5$ & 12.43 & 1.66 & 14.09 & 0.15 & 0.031 & 0.18 & $14.27(95.8 \%)$ & 98.7 & 88.2 & 11.8 \\
\hline$\eta_{j}>1$ & 11.90 & 1.50 & 13.40 & 0.065 & & 0.076 & $13.48(90.5 \%)$ & 99.4 & 88.8 & 11.2 \\
\hline$\eta_{j}>1.5$ & 11.02 & 1.28 & 12.30 & 0.026 & 0.0033 & 0.029 & $12.33(82.8 \%)$ & 99.8 & 89.6 & 10.4 \\
\hline$\eta_{j}>2$ & 9.69 & 0.99 & 10.68 & 0.0093 & 0.00086 & 0.010 & $10.69(71.8 \%)$ & 99.9 & 90.7 & 9.3 \\
\hline
\end{tabular}

Table 1. Cross section of $t h j$ production events with cuts on $\eta_{j}$. Contributions of the subprocesses $(u+c) b \rightarrow t h j,(\bar{d}+\bar{s}) b \rightarrow t h j$ and their sum are shown after each cut. The fraction of the events with the $b$-quark momentum along the negative $z$ direction is given as purity. Percent values in parenthesis show the fraction of thj events which survive after the cut.

\begin{tabular}{|c|c|c|c|c|c|c|c|c|c|c|}
\hline \multirow[t]{2}{*}{ Cut } & \multicolumn{3}{|c|}{$\sigma(q \bar{b} \rightarrow \bar{t} h j)[\mathrm{fb}]$} & \multicolumn{3}{|c|}{$\sigma(\bar{b} q \rightarrow \bar{t} h j)[\mathrm{fb}]$} & \multirow[t]{2}{*}{$\sigma(\bar{t} h j)[\mathrm{fb}]$} & \multirow{2}{*}{$\begin{array}{c}\text { Purity }[\%] \\
q \bar{b}\end{array}$} & \multicolumn{2}{|c|}{ Fraction in $q \bar{b}[\%]$} \\
\hline & $d \bar{b}+s \bar{b}$ & $\bar{u} \bar{b}+\bar{c} \bar{b}$ & Sum & $\bar{b} \bar{u}+\bar{b} \bar{c}$ & $\bar{b} d+\bar{b} s$ & Sum & & & $d \bar{b}+s \bar{b}$ & $\bar{u} \bar{b}+\bar{c} \bar{b}$ \\
\hline$\eta_{j}>0$ & 5.71 & 1.47 & 7.18 & 0.10 & 0.16 & 0.26 & 7.44 & 96.5 & 79.5 & 20.5 \\
\hline$\eta_{j}>0.5$ & 5.58 & 1.36 & 6.94 & 0.038 & 0.085 & 0.12 & $7.06(94.9 \%)$ & 98.3 & 80.4 & 19.6 \\
\hline$\eta_{j}>1$ & 5.32 & 1.19 & 6.51 & 0.013 & 0.041 & 0.054 & $6.56(88.2 \%)$ & 99.2 & 81.7 & 18.3 \\
\hline$\eta_{j}>1.5$ & 4.88 & 0.97 & 5.85 & 0.0039 & 0.017 & 0.021 & $5.87(78.9 \%)$ & 99.7 & 83.4 & 16.6 \\
\hline$\eta_{j}>2$ & 4.21 & 0.73 & 4.94 & 0.00099 & 0.0065 & 0.0075 & $4.95(66.5 \%)$ & 99.8 & 85.2 & 14.8 \\
\hline
\end{tabular}

Table 2. Cross section of $\bar{t} h j$ production events with cuts on $\eta_{j}$. Contributions of the subprocesses $(d+s) \bar{b} \rightarrow \bar{t} h j,(\bar{u}+\bar{c}) \bar{b} \rightarrow \bar{t} h j$ and their sum are shown after each cut. The fraction of the events with the $\bar{b}$-quark momentum along the negative $z$ direction is given as purity. Percent values in parenthesis show the fraction of $\bar{t} h j$ events which survive after the cut.

we estimate the purity by using the LO kinematical distributions at the parton level, and hence the numerical values quoted in tables 1 and 2 should be affected by QCD radiative corrections and by possible background contributions. Needless to say, events with $\eta_{j}<-1$ have exactly the same signal with those with $\eta_{j}>1$ because there is no distinction between the two colliding proton beam. From tables 1 and 2 , we find that the selection cut $\left|\eta_{j}\right|>1$ allow us to study $90 \%$ of $t h j$ and $88 \%$ of $\bar{t} h j$ events with full kinemetical reconstruction. In the following analysis, we assume that a significant fraction of $h$ and single $t$ or $\bar{t}$ production via $t$-channel $W$ exchange can be kinematically reconstructed, and define observables whose properties are directly determined by the helicity amplitudes of section 2 .

\subsection{Q and W distributions}

The differential cross section for the subprocess $u b \rightarrow d t h$ is

$$
d \hat{\sigma}=\frac{1}{2 \hat{s}} \frac{1}{4}\left(\left|\mathcal{M}_{+}\right|^{2}+\left|\mathcal{M}_{-}\right|^{2}\right) d \Phi_{3}(t h j)
$$

in terms of the helicity amplitudes $\mathcal{M}_{\sigma}$ in eq. (2.24), where $\hat{s}=\left(p_{u}+p_{b}\right)^{2}=\left(p_{d}+p_{t}+p_{h}\right)^{2}$, $1 / 4$ is the probability to find left-handed $u$ and $b$ quarks inside their PDFs, the color factor is unity for $t$-channel color-singlet exchange between the colliding quarks. The three-body 

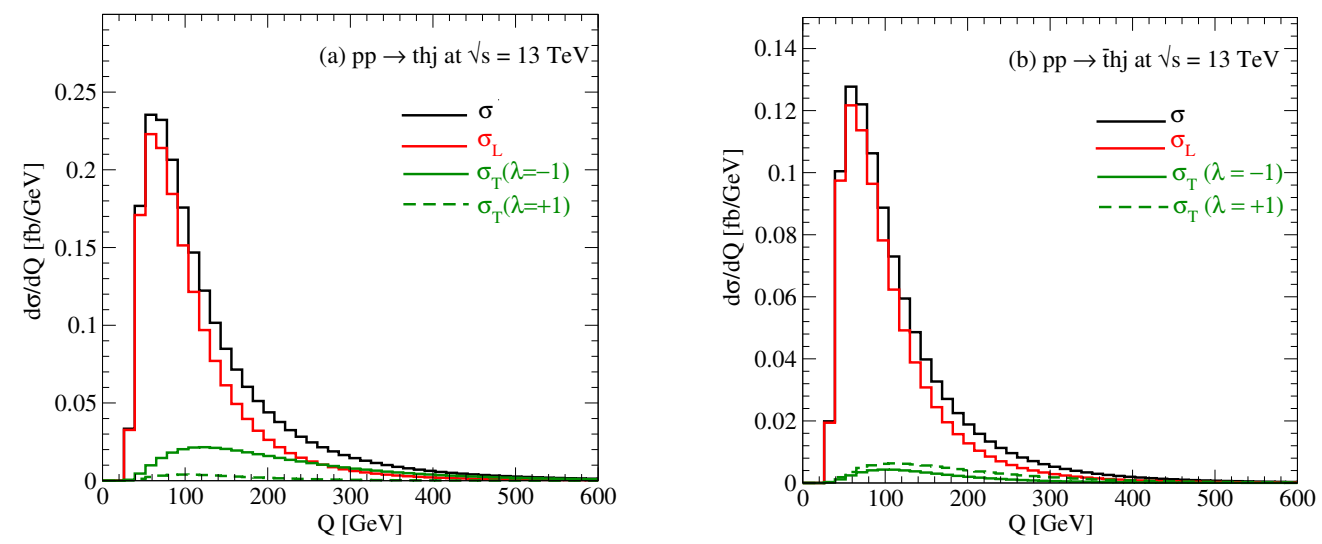

Figure 6. $d \sigma / d \mathrm{Q}$ for $p p>t h j$ (a) and $p p>\bar{t} h j$ in $p p$ collisions at $\sqrt{s}=13 \mathrm{TeV}$, with the jet tag condition of $p_{T}^{j}>30 \mathrm{GeV}$ and $1<\left|\eta_{j}\right|<4.5 . \mathrm{Q}=\sqrt{-q^{2}}$ is the invariant momentum transfer of the virtual $W^{+}$(a) or $W^{-}$(b). The red curves show contributions of the longitudinal $W$ with helicity $\lambda=0$, while the green curves show those of the transverse $W$ with helicity $\lambda= \pm 1$.

phase space $\mathrm{d} \Phi_{3}(t h j)$ is parametraized in the colliding parton c.m. frame, and then is expressed in terms of the kinematical variables in the $W^{+} b \rightarrow$ th rest frame eq. (2.11) and those of the Breit frame eqs. (2.12), (2.13), as depicted in figure 3. Kinematical details are summrized in appendix $\mathrm{C}$.

We show in figure 6 the distributions with respect to the momentum transfer Q, eq. (C.5). Contributions from $W^{\prime} s$ with helicity $\lambda=0\left(\sigma_{L}\right)$ and $\lambda= \pm 1\left(\sigma_{T}\right)$ separately and their sum are shown. Because the momentum transfer $\mathrm{Q}$ does not depend on the azimuthal angle, integration over $\phi$ about the $W$-momentum axis (the common $z$-axis in figure 3) projects out the $W$ helicity states and the interference among different $\lambda$ contributions vanish. It is clearly seen that the longitudinal $W$ with helicity $\lambda=0$ contribution in red solid curves dominates at small $\mathrm{Q}(\mathrm{Q} \lesssim 100 \mathrm{GeV})$ both for $t h j$ and $\bar{t} h j$. This is a consequence of the $\mathrm{W} / \mathrm{Q}$ enhancement of the $\lambda=0$ amplitudes as shown explicitly in eqs. (2.24) for $t h j$, and in eq. (2.39) for $\bar{t} h j$. Among the transverse $W$ contributions, $\lambda=-1$ (solid green) dominates over $\lambda=+1$ (dashed green) for $t h j$, but they are comparable for $\bar{t} h j$.

This somewhat different behaviour of the transverse $W$ contribution between thj and $\bar{t} h j$ processes needs clarification, and we show in figure 7 the distribution with respect to $\mathrm{W}$, the invariant mass of the $t h$ system. The upper plots (a) and (b) are for $\mathrm{Q}<100 \mathrm{GeV}$, and the lower plots (c) and (d) are for $\mathrm{Q}>100 \mathrm{GeV}$. The left figures (a) and (c) are for $t h j$, while the right ones (b) and (d) are for $\bar{t} h j$. Again contributions from the three helicity states of the exchanged $W$ are shown separately. It is clearly seen that at small $\mathbf{Q}(\mathbf{Q}<100 \mathrm{GeV})$ and large $\mathrm{W}, \mathrm{W} \gtrsim 500 \mathrm{GeV}$, the longitudinal $W(\lambda=0)$ contribution dominates the cross sections of both thj (a) and $\bar{t} h j$ (b) production. The transverse $W$ contributions are significant at large $\mathbf{Q}(\mathbf{Q}>100 \mathrm{GeV})$, where the left handed $W$ with helicity $\lambda=-1$ dominates over the longitudinal $W$ with helicity $\lambda=0$ at $\mathrm{W} \lesssim 400 \mathrm{GeV}$ for $t h j$.

On the other hand, the right-handed $W^{-}$dominates $\bar{t} h j$ production at small $\mathrm{W}$, especially at large $Q(Q>100) \mathrm{GeV}$, see figure $7(\mathrm{~d})$. This is because the $W^{-}$with helicity $\lambda=+1$ collides with the right-handed $\bar{b}$-quark, giving $J_{z}=+\frac{1}{2}$ initial state with no $\bar{\beta}$ 

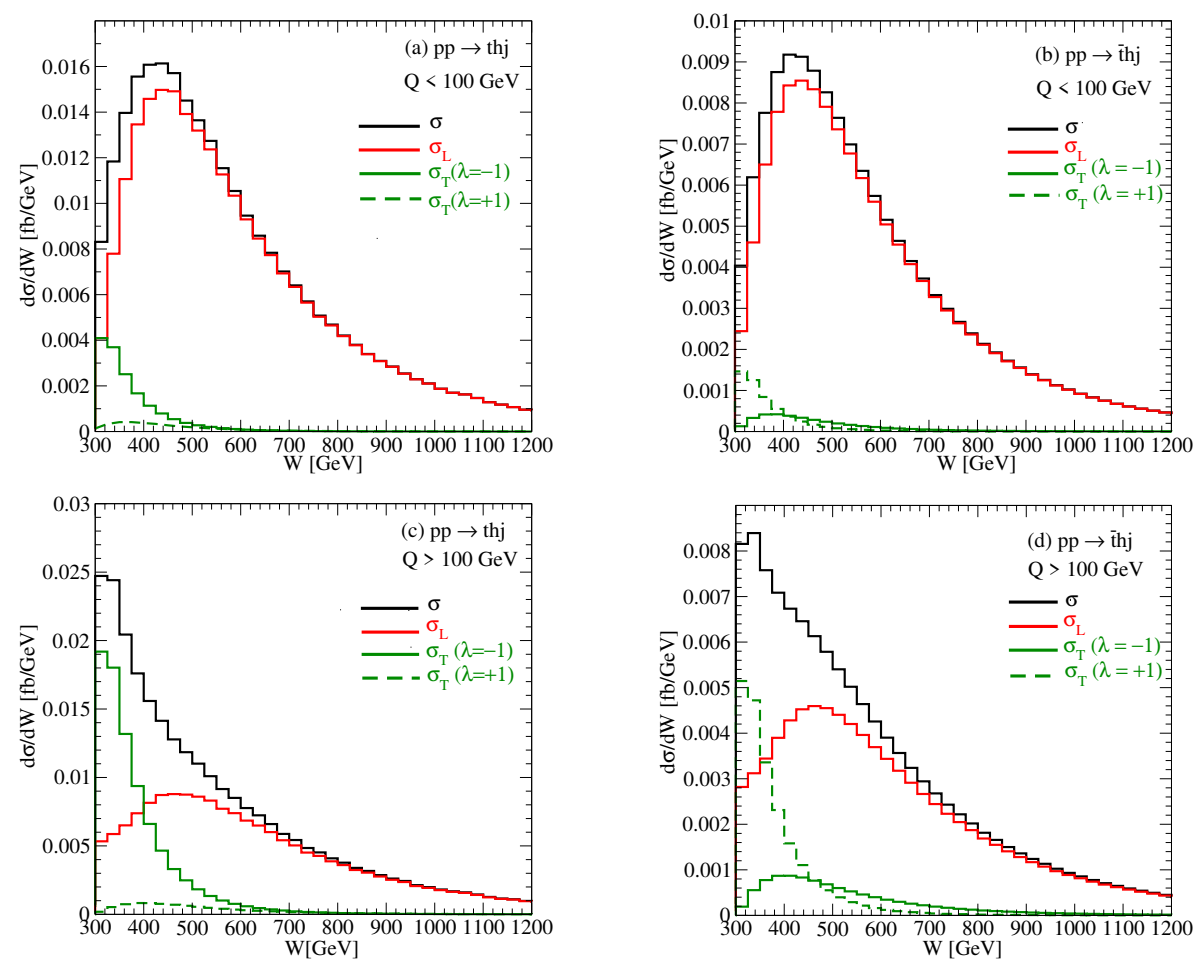

Figure 7. $d \sigma / d \mathrm{~W}$ v.s. $\mathrm{W}$, where $\mathrm{W}=\sqrt{P_{t h}^{2}}=m(t h)$ is the invariant mass of $t h$ system. The upper two plots are for small $Q(Q<100 \mathrm{GeV})$, while the lower two plots are for large $Q(Q>100 \mathrm{GeV})$. The left plots (a) and (c) are for $t h j$, while the right ones (b) and (d) are for $\bar{t} h j$. The red curves show contributions of the longitudinal $W$ with helicity $\lambda=0$, while the green curves show those of the transverse $W$ with helicity $\lambda= \pm 1$.

suppression, as can be seen from the first terms in eqs. (2.39a) and (2.39b). The $W^{-}$with helicity $\lambda=-1$ contribution dominates over $\lambda=+1$ at large $\mathrm{W}$, because the left-handed $d$-quark prefers to emit $W^{-}$with helicity $\lambda=-1$, as can be seen from the $d \rightarrow u W^{-}$ splitting amplitudes $J_{\lambda}$, eq. (2.15).

Summing up, the $W^{-}$with helicity $\lambda=+1$ contribution is significant near the threshold ( $\mathrm{W} \lesssim 400 \mathrm{GeV}$ ) for $\bar{t} h j$ production, while the $W^{-}$with helicity $\lambda=-1$ contribution takes over at larger $\mathrm{W}$ because of dominant $d$-quark contribution. In contrast, for the thj production, the $\lambda=+1$ contribution (green dashed lines) is deeply suppressed, as the disfavored helicity emitted from left-handed $u$ quark at large $\mathrm{W}$ and by the $p$-wave threshold suppression at small W, making them very small both at small (a) and large $\mathbf{Q}(\mathrm{b})$.

\section{Azimuthal angle asymmety}

In figure 8(a), we show distributions of the azimuthal angle between the emission $\left(u \rightarrow d W^{+}\right.$, $\bar{d} \rightarrow \bar{u} W^{+}$, etc.) plane and the $W^{+} b \rightarrow t h$ production plane about the common $W^{+}$ momentum direction in the $W^{+} b$ rest frame; see figure 3. Shown in figure 8(b) are the same distributions for $p p \rightarrow \bar{t} h j$ process, where the azimuthal angle is between the $W^{-}$ emission plane and the $W^{-} \bar{b} \rightarrow \bar{t} h$ production plane about the common $W^{-}$momentum 

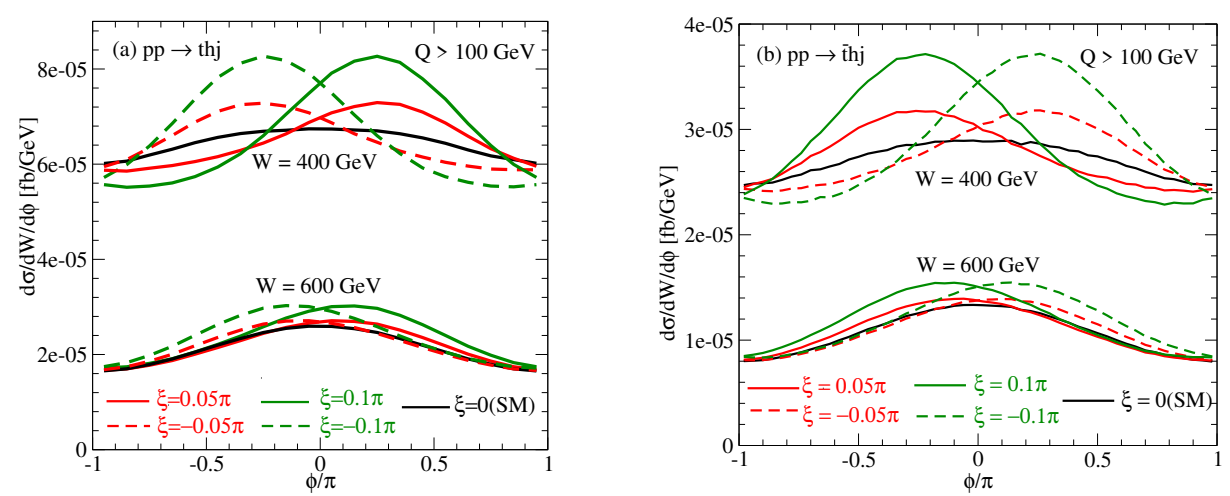

Figure 8. $d \sigma / d \mathrm{~W} / d \phi$ v.s. $\phi$ at $\mathrm{W}=400$ and $600 \mathrm{GeV}$ for $\mathrm{Q}>100 \mathrm{GeV}$ for $p p \rightarrow$ thj (a) and $\bar{t} h j$ (b). Black, red and green curves are for the SM $(\xi=0), \xi= \pm 0.05 \pi$, and $\pm 0.1 \pi$. The solid curve are for $\xi \geq 0$, while the dashed curves are for $\xi<0$.
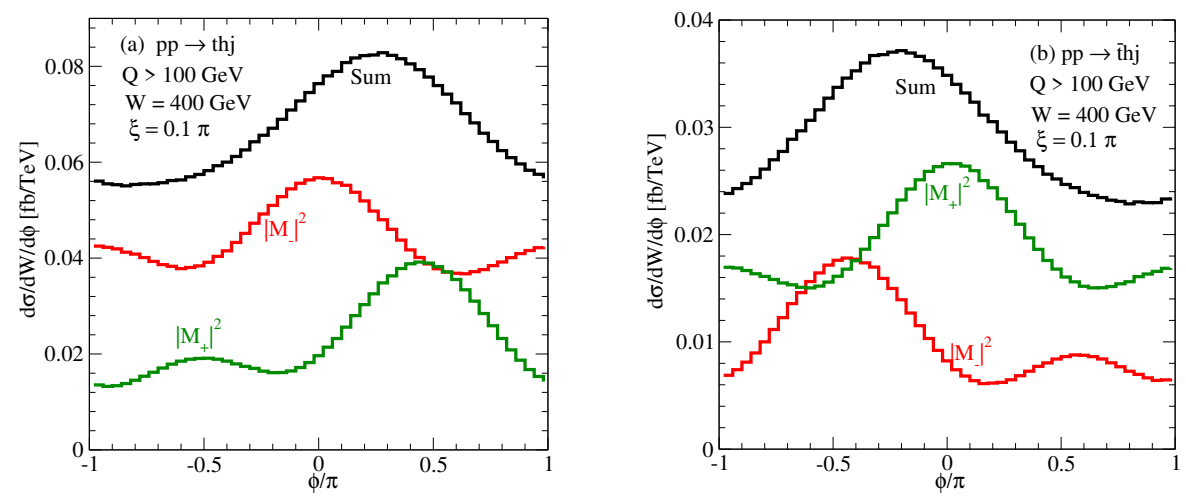

Figure 9. Azimuthal angle distribution of $\left|\mathcal{M}_{+}^{2}\right|$ (red) and $\left|\mathcal{M}_{-}^{2}\right|$ (green) and the sum (black) for $p p \rightarrow t h j$ (a) and $p p \rightarrow \bar{t} h j$ (b) for $\mathrm{Q}>100 \mathrm{GeV}$ events at $\mathrm{W}=400 \mathrm{GeV}$, for $\xi=0.1 \pi$.

direction. The results are shown at $\mathrm{W}=400$ and $600 \mathrm{GeV}$ for large $\mathrm{Q}(\mathrm{Q}>100 \mathrm{GeV})$. The black, red and green curves are for the SM $(\xi=0), \xi= \pm 0.05 \pi$, and $\pm 0.1 \pi$, respectively. Solid curves are for $\xi \geq 0$ while dashed curves are for $\xi<0$. The $\phi$ distributions are proportional to

$$
\left|\mathcal{M}_{+}\right|^{2}+\left|\mathcal{M}_{-}\right|^{2}
$$

where the top polarization is summed over. Likewise, they are proportional to $\left|\overline{\mathcal{M}}_{+}\right|^{2}+\left|\overline{\mathcal{M}}_{-}\right|^{2}$ for $\bar{t} h j$ events. Analytic expression for the amplitudes, $\mathcal{M}_{ \pm}$and $\overline{\mathcal{M}}_{ \pm}$ are given in eqs. (2.24) and (2.39), respectively, where we can tell that azimuthal angle dependences are in the transversely polarized $W$ exchange amplitudes. The asymmetry is large at small $\mathrm{W}$ and large $\mathrm{Q}$ because the transverse $W^{ \pm}$amplitudes are significant there, see figure 7 . The asymmetry remains significant at $\mathrm{W}=400 \mathrm{GeV}$, however, even for events with $\mathrm{Q}<100 \mathrm{GeV}[32]$.

We show in figure 9(a) the azimuthal angle distribution of right-handed and lefthanded top quark separately, in green and red curves respectively, at $\mathrm{W}=400 \mathrm{GeV}$ for events with $\mathrm{Q}>100 \mathrm{GeV}$ and $\xi=0.1 \pi$. Their sum, given by the black curve agree with the 


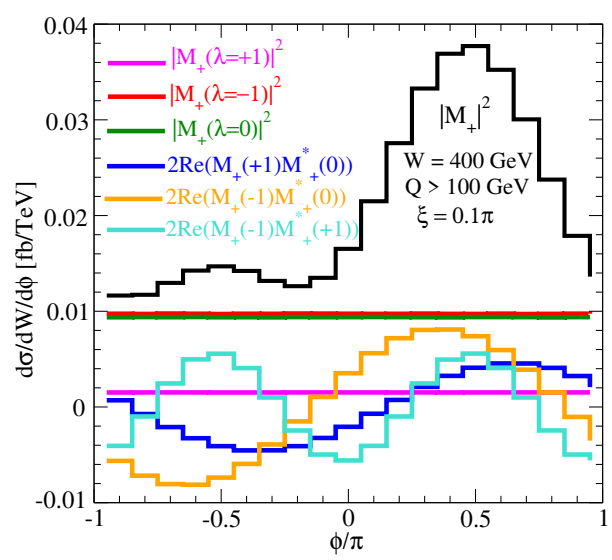

Figure 10. Azimuthal angle distribution of $\left|\mathcal{M}_{+}\right|^{2}=\left|\sum_{\lambda} \mathcal{M}_{+}(\lambda)\right|^{2}$ in terms of the six combinations of the three $W^{+}$polarization amplitudes, $\mathcal{M}_{+}(\lambda)$ for $\lambda=+1,-1$, and 0 . The contribution of the helicity $+1 / 2$ top quark production in the subprocess $u b \rightarrow d t h$ is shown.

corresponding curve in figure 8(a). As expected from the analytic expressions eqs. (2.24) and (2.27), $\left|\mathcal{M}_{-}\right|^{2}$ is almost symmetric about $\phi=0$, and the asymmetry is mainly from $\left|\mathcal{M}_{+}\right|^{2}$. Likewise, for the $\bar{t} h j$ events, shown in figure 9(b), the asymmetry is mainly from left handed $\bar{t}$ quark, depicted by the red $\left|\overline{\mathcal{M}}_{-}\right|^{2}$ curve.

The origin of the azimuthal angle asymmetry comes from the interference between transverse $W$ amplitudes with the $e^{ \pm i \phi}$ phase factor for $W$ with helicity $\lambda= \pm 1$ and the longitudinal $W$ with helicity $\lambda=0$ amplitudes as shown in eq. (2.24) for $u b \rightarrow$ thj and eq. (2.39) for $d \bar{b} \rightarrow u \bar{t} h$. We show in figure 10 the azimuthal angle distribution of $\left|\mathcal{M}_{+}\right|^{2}$ for the subprocess $u b \rightarrow d t h$, together with the six individual contributions

$$
\begin{aligned}
\left|\mathcal{M}_{+}\right|^{2}= & \left|\sum_{\lambda} \mathcal{M}_{+}(\lambda)\right|^{2} \\
= & \left|\mathcal{M}_{+}(+1)\right|^{2}+\left|\mathcal{M}_{+}(-1)\right|^{2}+\left|\mathcal{M}_{+}(0)\right|^{2} \\
& +2 \operatorname{Re} \mathcal{M}_{+}(+1) \mathcal{M}_{+}^{*}(0)+2 \operatorname{Re} \mathcal{M}_{+}(-1) \mathcal{M}_{+}^{*}(0)+2 \operatorname{Re} \mathcal{M}_{+}(-1) \mathcal{M}_{+}^{*}(+1),
\end{aligned}
$$

separately. The three squared terms, $\left|\mathcal{M}_{+}(\lambda)\right|^{2}$, for $\lambda=+1,-1$ and 0 , give no $\phi$ dependence, while the interference terms between $\mathcal{M}_{+}(0)$ and $\mathcal{M}_{+}(-1)$ amplitudes give terms proportional to $\sin \phi \sin \xi$ with positive coefficients, leading to positive $\langle\sin \phi\rangle$ for $\sin \xi>0$. It is clearly seen from figure 10 that $\left|\mathcal{M}_{+}(\lambda=-1)\right|^{2} \simeq\left|\mathcal{M}_{+}(\lambda=0)\right|^{2} \gg\left|\mathcal{M}_{-}(\lambda=+1)\right|^{2}$ at $\mathrm{W}=400 \mathrm{GeV}$ for $\mathrm{Q}>100 \mathrm{GeV}$ for the subprocess $u b \rightarrow d t h$, consistent with the trend expected from the SM amplitudes at $\xi=0$, shown in figure $7(\mathrm{c})$. It is therefore the interference between the $\mathcal{M}_{+}(\lambda=-1)$ and $\mathcal{M}_{+}(\lambda=0)$ amplitudes, shown by the orange curve in figure 10, which determines the asymmetry $\langle\sin \phi\rangle$. The interference between the $W$ with helicity $\lambda= \pm 1$ exchange amplitudes give terms of the form $\sin 2 \phi \sin \xi$, which gives rise to another asymmetry $\langle\sin 2 \phi\rangle$. Because $\left|\mathcal{M}_{-}(\lambda=+1)\right|$ is generally small at all $\mathbf{Q}$ and W regions, as shown in figures $7(\mathrm{a})$ and (c), the asymmetry $\langle\sin 2 \phi\rangle$ turns out to be small in our analysis. We therefore do not show results on $\langle\sin 2 \phi\rangle$ in the following, but note that its measurement should improve the $\xi$ sensitivity at a quantitive level, and that it should 


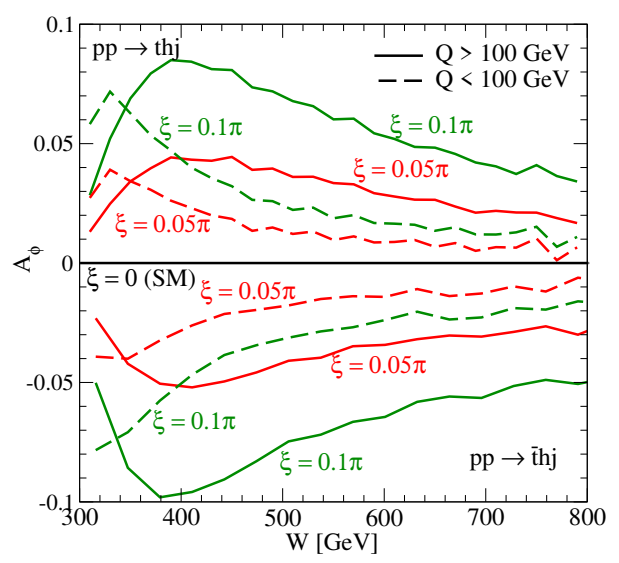

Figure 11. Asymmetry $A_{\phi}(\mathrm{W})$ for $p p \rightarrow t h j$ and $p p \rightarrow \bar{t} h j$ as functions of $\mathrm{W}$, the invariant mass of $t h$ or $\bar{t} h$ system. Large $\mathbf{Q}(\mathbf{Q}>100 \mathrm{GeV})$ events are shown by solid lines, while small $\mathbf{Q}(\mathbf{Q}<100) \mathrm{GeV}$, events are shown by dashed curves. Results are shown for $\xi=0$ (SM), $\xi=0.05 \pi$ (red) and $0.1 \pi$ (green). $A_{\phi}>0$ for $t h$ and $A_{\phi}<0$ for $\bar{t} h$, when $\xi>0$.

be sensitive to other type of new physics that affects mainly the transversally polarized $W$ amplitudes. It may be worth noting that asymmetry $\langle\sin 2 \phi\rangle$ is larger in $\bar{t} h j$ process, because both $\lambda= \pm 1$ transversally polarized $W$ contributions are significant, as can be seen from figure $7(\mathrm{~d})$, especially at large $\mathrm{Q}$ and small $\mathrm{W}$.

In figure 11, we show the azimuthal asymmetry integrated over $\phi$,

$$
A_{\phi}(\mathrm{W})=\frac{\int_{-\pi}^{\pi} d \phi \operatorname{sgn}(\phi) d \sigma / d \mathrm{~W} / d \phi}{d \sigma / d \mathrm{~W}}
$$

as a function of the invariant mass $\mathrm{W}$ of the $t h$ or $\bar{t} h$ system for $\xi=0(\mathrm{SM}), \pm 0.05 \pi$ (red curve) and $\pm 0.1 \pi$ (green curve). The asymmetry for large $Q(Q>100) \mathrm{GeV}$ events is shown by solid curves, while those for small $Q(Q<100 \mathrm{GeV})$ is shown by dashed curves. The positive aysmmetry is found for thj events, while negative asymmetry is found for $\bar{t} h j$, in accordance with the observation from the $\phi$ distribution in figure 8. Generally speaking, the asymmetry is large for large $Q$ events at around $W \sim 400 \mathrm{GeV}$ where the magnitudes of the transverse and longitudinal $W$ exchange amplitudes are comparable in figure 7 (c) and $(\mathrm{d})$. For small $\mathrm{Q},(\mathrm{Q}<100 \mathrm{GeV})$, the asymmetry is significant only near the threshold, $\mathrm{W} \sim m_{t}+m_{h}$, where the transverse $W$ amplitudes are non-negligible in figure $7(\mathrm{a})$ and (b).

Because the asymmetry due to the term linear in $\sin \xi$ are nearly absent in $\left|\mathcal{M}_{-}\right|^{2}$ for $t h j$ and in $\left|\overline{\mathcal{M}}_{+}\right|^{2}$ for $\bar{t} h j$, as can be seen from eqs. (2.24b) and (2.39a) for $\delta \simeq \delta^{\prime}$ approximation, we can expect enhancement of the asymmetry by selecting right-handed top and the left-handed anti-top. This can easily be achieved when $t$ and $\bar{t}$ decay semileptonically, where the charged-lepton decay angular distribution in the $t$ or $\bar{t}$ rest frame takes the form [39]

$$
\begin{aligned}
& \frac{d \Gamma(t \rightarrow b \bar{\ell} \nu)}{d \cos \theta_{\bar{\ell}}} \sim\left(1+\sigma \cos \theta_{\bar{\ell}}\right)^{2}, \\
& \frac{d \Gamma(\bar{t} \rightarrow \bar{b} \ell \bar{\nu})}{d \cos \theta_{\ell}} \sim\left(1-\bar{\sigma} \cos \theta_{\ell}\right)^{2},
\end{aligned}
$$




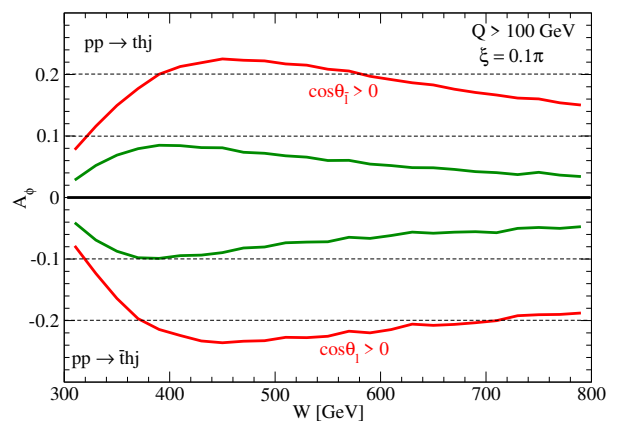

Figure 12. Asymmetry $A_{\phi}(\mathrm{W})$ as a function of invariant mass $\mathrm{W}$ of the $t h$ or $\bar{t} h$ system in $p p \rightarrow t h j$, or $\bar{t} h j$ process, when the momentum transfer $Q$ of the exchanged $W$ is larger than $100 \mathrm{GeV}$. $A_{\phi}(\mathrm{W})=0$ for the SM $(\xi=0)$. $A_{\phi}(\mathrm{W})>0$ for $t h j$, while $A_{\phi}(\mathrm{W})<0$ for $\bar{t} h j$ when $\xi=0.1 \pi$. The green solid curves are the asymmetries without cuts. The asymmetry grows to the red curves when the charged lepton decay angle satisfy $\cos \theta_{\bar{\ell}}, \cos \theta_{\ell}>0$ in the $t$ and $\bar{t}$ rest frame.

about the helicity axis, where $\sigma$ and $\bar{\sigma}$ are twice the helicities of $t$ and $\bar{t}$, respectively, in the $t h$ or $\bar{t} h$ rest frame. For instance, if we select those events with

$$
\cos \theta_{\bar{\ell}}, \cos \theta_{\ell}>0
$$

then $d \sigma / d \mathrm{~W} / d \phi$ is proportional to

$$
\begin{aligned}
& \frac{7}{8}\left|\mathcal{M}_{+}\right|^{2}+\frac{1}{8}\left|\mathcal{M}_{-}\right|^{2} \quad \text { for top } \\
& \frac{1}{8}\left|\overline{\mathcal{M}}_{+}\right|^{2}+\frac{7}{8}\left|\overline{\mathcal{M}}_{-}\right|^{2} \quad \text { for anti-top, }
\end{aligned}
$$

and the asymmetry is significantly larger, as shown in figure 12 for $\xi=0.1 \pi$ when $Q>100 \mathrm{GeV}$. The asymmetries shown by the green curves are when no cuts are applied, and they agree with the corresponding curves in figure 11. The asymmetry grows to $A_{\phi}(\mathrm{W}) \sim 0.22$ for $t h j$ events and $A_{\phi}(\mathrm{W}) \sim-0.23$ for $\bar{t} h j$ events, both at around $\mathrm{W} \sim 450 \mathrm{GeV}$ with the selection cut of the $t$ and $\bar{t}$ decay charged lepton angles in eq. (4.5). We note here that the charged leptons from $t$ and $\bar{t}$ decays are isolated from the tagged jet because the jet pseudo rapidity tends to be rather large $\left|\eta_{j}\right| \gtrsim 2$, see e.g. figure 5 , while the leptons and $b$-jets from $t / \bar{t}$ and $h$ tend to have small pseudo rapidity.

Since the statistical error of the asymmetry scales as $\sqrt{\left(1-A_{\phi}^{2}\right) / N}$ for $N$ events used in the analysis, the number of thj and $\bar{t} h j$ events which can be used to measure the asymmetry $A_{\phi}$ in figures 11 and 12 determines the experimental feasibility of measuring non-zero CP phase $\xi$. Because the azimuthal asymmetry $A_{\phi}$ has opposite sign between top and anti-top, we should select events where they decay semi-leptonically into charged $e$ or $\mu$. If the Higgs boson can be identified in the di- $b$ jet mode, we can hope to study 10,000 useful events at HL-LHC, and the asymmetries can be measured at a percent level. On the other hand, if the background is high for the di- $b$ mode, the number of useful events may reduce to 100, giving the statistical error of the order of $10 \%$. Dedicated background studies for $h \rightarrow b b$ mode in the $t h j$ and $\bar{t} h j$ events are eagerly waited. 


\section{Polarization asymmetries}

We are now ready to discuss the polarization of the top quark in the single top $+h$ production processes. We first note that the helicity amplitudes $\mathcal{M}_{+}$and $\mathcal{M}_{-}$in eq. (2.24) for the subprocess $u b \rightarrow d t h$, and those in eq. (2.39) for $d \bar{b} \rightarrow u \bar{t} h$ are purely complex numbers when production kinematics $\left(\sqrt{\hat{s}}, \mathrm{Q}, \mathrm{W}, \cos \tilde{\theta}, \cos \theta^{*}, \phi\right)$ are fixed. This is a peculiar feature of the SM where only the left-handed $u, d$, and $b$ quarks, and their anti-particles with right-handed helicities contribute to the single $t$ and $\bar{t}$ production process via $W$ exchange. It implies that the produced top quark polarization state is expressed as the superposition

$$
|t\rangle=\frac{\mathcal{M}_{+}\left|J_{z}=+\frac{1}{2}\right\rangle+\mathcal{M}_{-}\left|J_{z}=-\frac{1}{2}\right\rangle}{\sqrt{\left|\mathcal{M}_{+}\right|^{2}+\left|\mathcal{M}_{-}\right|^{2}}}
$$

in the top quark rest frame, where the quantization axis is along the top momentum direction in the th rest frame, where the top quark helicity is defined. The top quark is hence in the pure quantum state with $100 \%$ polarization, with its orientation fixed by the complex number $\mathcal{M}_{-} / \mathcal{M}_{+}$. Its magnitude $\left|\mathcal{M}_{-} / \mathcal{M}_{+}\right|$determines the polar angle and the phase $\arg \left(\mathcal{M}_{-} / \mathcal{M}_{+}\right)$determines the azimuthal angle of the top spin direction. ${ }^{2}$ Therefore, the kinematics dependence of the polarization direction can be exploited to measure the CP phase $\xi$, e.g. by combining matrix element methods with the polarized top decay density matrix. ${ }^{3}$ Exactly the same applies for the $\bar{t}$ spin polarization, whose quantum state can be expressed as in eq. (5.1) where the helicity amplitudes $\mathcal{M}_{ \pm}$are replaced by $\overline{\mathcal{M}}_{ \pm}$.

In this report, we investigate the prospects of studying $\mathrm{CP}$ violation in the htt coupling through the top and anti-top quark polarization asymmetries in the single $t h$ and $\bar{t} h$ processes respectively, with partial integration over the final state phase space. For this purpose, we introduce a complex matrix distribution

$$
d \sigma_{\lambda \lambda^{\prime}}=\int d x_{1} \int d x_{2}\left[D_{u}\left(x_{1}, \mu\right) D_{b}\left(x_{2}, \mu\right)+D_{b}\left(x_{1}, \mu\right) D_{u}\left(x_{2}, \mu\right)\right] \frac{1}{2 \hat{s}} \frac{1}{4} M_{\lambda} M_{\lambda^{\prime}}^{*} d \Phi_{3}(d t h)
$$

Note that the matrix (5.2) is normalized such that its trace gives the differential cross section of eq. (3.1).

$$
d \sigma=d \sigma_{++}+d \sigma_{--}
$$

Here we denote $\lambda / 2$ and $\lambda^{\prime} / 2$ for the top helicity, and the $1 / 4$ factor accounts for the colliding parton spin average, just as in eq. (3.6) for the subprocess cross section. All the other subprocesses which contribute to the same $t h j$ final state, $c b \rightarrow s t h, \bar{d} b \rightarrow \bar{u} t h$, $\bar{s} b \rightarrow \bar{c} t h$, whose matrix elements are given in eqs. (2.29) and (2.30) should be summed over in the matrix (5.2). The integration over phase space and the summation over different subprocess contribution make the top quark in the mixed state and its polarization density matrix is given by

$$
\rho_{\lambda \lambda^{\prime}}=\frac{d \sigma_{\lambda \lambda^{\prime}}}{d \sigma_{++}+d \sigma_{--}}=\frac{1}{2}\left[\delta_{\lambda \lambda^{\prime}}+\sum_{k=1}^{3} P_{k} \sigma_{\lambda \lambda^{\prime}}^{k}\right]
$$

for an arbitrary distribution

\footnotetext{
${ }^{2}$ See appendix A for a pedagogical review of quantum mechanics.

${ }^{3}$ The top quark decay polarization density matrices for its semi-leptonic and hadronic decays are given in appendix B.
} 
The coefficients of the three $\sigma$ matrices makes a three-vector, $\vec{P}=\left(P_{1}, P_{2}, P_{3}\right)$, whose magnitude $P=|\vec{P}|$ gives the degree of polarization $(P=1$ for $100 \%$ polarization, $P=0$ for no polarization), while its spatial orientation gives the direction of the top quark spin in the top rest frame. The polarization vector $\vec{P}$ in (5.4) can be obtained directly from the matrix distribution (5.2) as follows

$$
\begin{aligned}
P_{1} & =\frac{2 \operatorname{Re} \int d \sigma_{+-}}{\int d \sigma_{++}+\int d \sigma_{--}}, \\
P_{2} & =\frac{-2 \operatorname{Im} \int d \sigma_{+-}}{\int d \sigma_{++}+\int d \sigma_{--}}, \\
P_{3} & =\frac{\int d \sigma_{++}-\int d \sigma_{--}}{\int d \sigma_{++}+\int d \sigma_{--}},
\end{aligned}
$$

where the integral over the phase space can be chosen appropriately in order to avoid possible cancellation of polarization asymmetries. For the helicity amplitudes (2.24) calculated in the $t h$ rest frame, the $z$-axis is along the top momentum in the th rest frame, and the $y$-axis is along the $\vec{q} \times \vec{p}_{t}$ direction, perpendicular to the $W^{+} b \rightarrow$ th scattering plane. In appendix A, we obtain the orientation of the top quark spin in terms of the helicity amplitudes for a pure state and for general mixed states.

The polarization of $\bar{t}$ quark is obtained also from the matrix distribution (5.2) with the $\bar{t} h j$ amplitudes $\overline{\mathcal{M}}_{\bar{\lambda}} \overline{\mathcal{M}}_{\bar{\lambda}^{\prime}}^{*}$, simply by replacing $\lambda \lambda^{\prime}$ by $\bar{\lambda} \bar{\lambda}^{\prime}$ in the density matrix (5.4). The orientation of the polarization vector is measured in the same frame, where the $z$-axis is now along the $\bar{t}$ quark momentum direction in the $\bar{t} h$ rest frame and the $y$-axis is along the $\vec{q} \times \overrightarrow{p_{\hat{t}}}$ direction.

We show in figure 13 the three components $\left(P_{1}, P_{2}, P_{3}\right)$ of the polarization vector $\vec{P}$ as a function of the top (anti-top) scattering angle $\cos \theta^{*}$ in the $t h(\bar{t} h)$ rest frame, at $\mathrm{W}=400 \mathrm{GeV}$ (upper four plots) and $600 \mathrm{GeV}$ (lower four plots), when all the other kinematical variables are integrated over subject to the constraint $\mathrm{Q}<100 \mathrm{GeV}$ (a), (e), (c), (g) and $\mathbf{Q}>100 \mathrm{GeV}(\mathrm{b}),(\mathrm{f}),(\mathrm{d}),(\mathrm{h})$. The left-hand side of figure 13 gives the top polarization in $t h j$ processes, while the right-hand side plots give the $\bar{t}$ polarization in $\bar{t} h j$ processes.

Let us first examine the top polarization in figure 13 (a), (b), (c), (d). It is the polarization perpendicular to the scattering plane,

$$
P_{2}=\left\langle\frac{\vec{q} \times \vec{p}_{t} \cdot \vec{P}}{\left|\vec{q} \times \vec{p}_{t}\right|}\right\rangle=\left\langle\frac{\vec{p}_{b} \times \vec{p}_{h} \cdot \vec{P}}{\left|\vec{p}_{b} \times \vec{p}_{h}\right|}\right\rangle
$$

which vanishes for $\xi=0$ in the SM in the tree-level, as shown by red solid curves in all the plots. $P_{1}, P_{2}$ and $P_{3}$ are given by blue, red and green curves, respectively. The three curves for each polarization components, $P_{k}(k=1,2,3)$, are for $\xi=0$ (solid curves), $\xi=0.05 \pi$ (dashed curves) and for $\xi=0.1 \pi$ (dot-dashed curve). Significant $P_{2}$ polarization is expected even for $\xi=0.05 \pi$ shown by red-dashed curves. The plots on the right side (e), (f), (g), (h) give $\bar{t}$ polarization in the anti-top and $h$ production events. In (a), (b), (c), (d), $P_{3} \approx-1$ (and hence $P_{1} \approx P_{2} \approx 0$ ) at $\cos \theta^{*}=-1$, because $\left|\mathcal{M}_{-}\right| \gg\left|\mathcal{M}_{+}\right|$at $\cos \frac{\theta^{*}}{2}=0$ and $\sin \frac{\theta^{*}}{2}=1$ in eq. (2.24) for $u b \rightarrow d t h$ and $c b \rightarrow$ sth, and similarly in eq. (2.30) for $\bar{d} b \rightarrow \bar{u} t h$ and $\bar{s} b \rightarrow \bar{c} t h$, which have the same $W^{+} b \rightarrow t h$ amplitudes. The $\lambda= \pm 1$ 

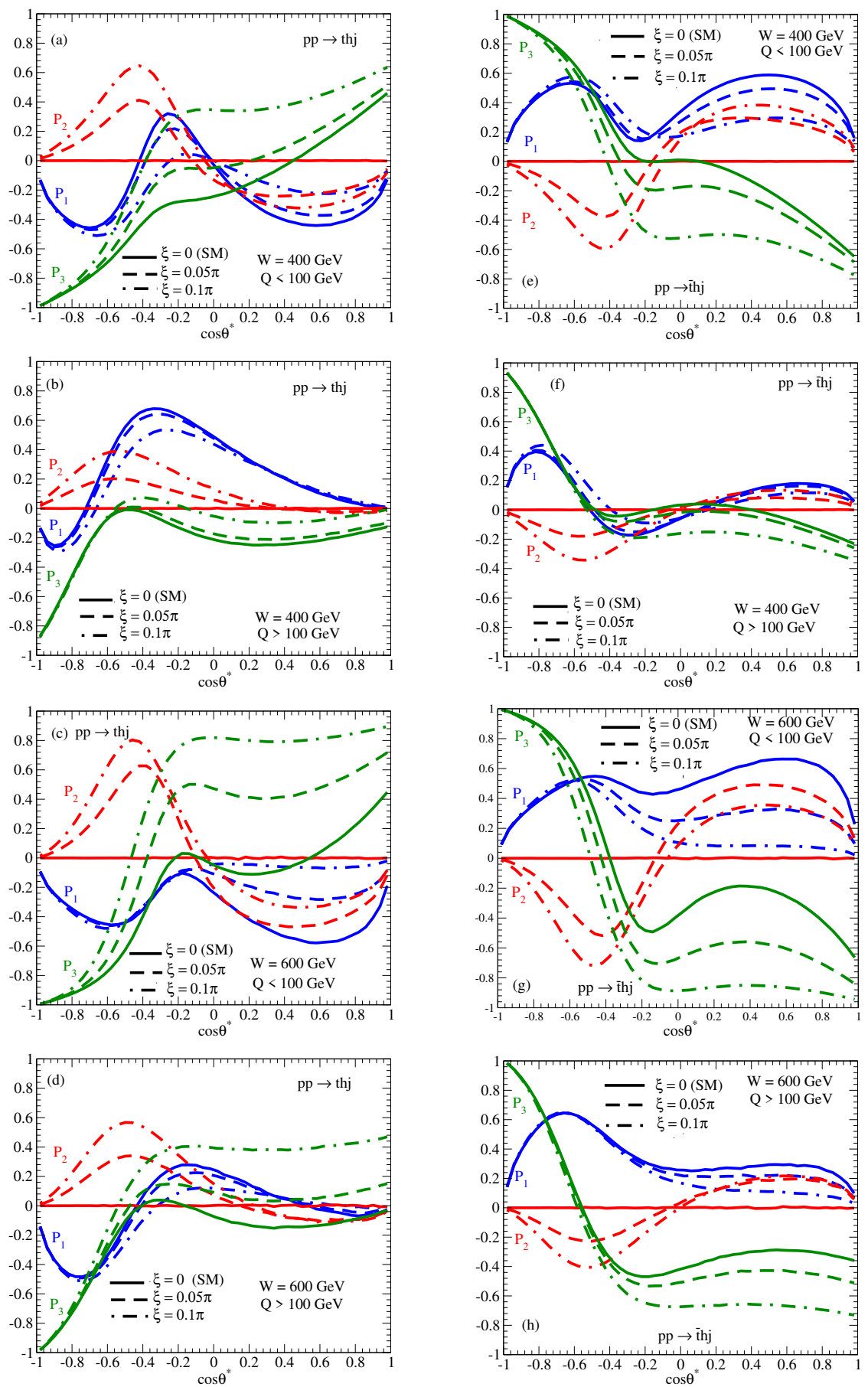

Figure 13. $t$ and $\bar{t}$ quark polarization vector $\vec{P}=\left(P_{1}, P_{2}, P_{3}\right)$ as function of $\cos \theta^{*}$, the $t$ or $\bar{t}$ scattering angle in the $W^{+} b \rightarrow t h\left(W^{-} \bar{b} \rightarrow \bar{t} h\right)$ scattering plane at $\mathrm{W}=400 \mathrm{GeV}, \mathrm{W}=600 \mathrm{GeV}$ for $\mathrm{Q}>100 \mathrm{GeV}$ and $\mathrm{Q}<100 \mathrm{GeV}$. The LO predictions are shown for the SM $(\xi=0)$ by solid curves, $\xi=0.05 \pi$ by dashed curves and for $\xi=0.1 \pi$ by dash-dotted curves. The left four panels (a) to (d) are for $p p \rightarrow t h j$ production and the right four panels (e) to (h) are for $p p \rightarrow \bar{t} h j$ production. $P_{2}$ (denoted by the red curves) is the polarization component perpendicular to the scattering plane. $P_{2}=0$ in the $\operatorname{SM}(\xi=0)$. 
amplitudes in $\mathcal{M}_{+}$are strongly suppressed at $\cos \theta^{*}=-1$, while the $\lambda=0$ amplitudes in $\mathcal{M}_{-}$are not only non-vanishing at $\cos \theta^{*}=-1$ but also $\mathrm{W} / \mathrm{Q}$ enhanced. The magnitude of $\mathcal{M}_{+}$grows quickly as $\cos \theta^{*}$ deviates from -1 , and the interference between $\mathcal{M}_{+}$and $\mathcal{M}_{-}$ gives nontrivial polarization of the top quark.

As $\cos \theta^{*}$ deviates from $-1, P_{3}$ deviates from -1 according to the growth of $\left|\mathcal{M}_{+}\right|^{2} /\left|\mathcal{M}_{-}\right|^{2}$, but $\left|P_{1}\right|$ (and also $\left|P_{2}\right|$ when $\xi \neq 0$ ) grows quickly as they are linear in $\mathcal{M}_{+}$. The polarization $P_{2}$ normal to the scattering plane can become as large as 0.6 even for $\xi=0.05 \pi$, when $\mathrm{Q}<100 \mathrm{GeV}$ at $\mathrm{W}=600 \mathrm{GeV}$; see figure $13(\mathrm{c})$. This is because at small $\mathbf{Q}$ and large $\mathrm{W}$, the longitudinal $W$ with helicity $\lambda=0$ contribution dominates over the transverse $W$ with helicity $\lambda= \pm 1$ contributions, and hence the integration over the azimuthal angle $\phi$ does not diminish much the degree of top polarization.

Likewise, the $\bar{t}$ polarization is shown in the right hand side of figure 13, for the same configuration of $\mathrm{W}=400 \mathrm{GeV}(e),(f)$ and $600 \mathrm{GeV}(g),(h)$, for $\mathrm{Q}<100 \mathrm{GeV}(e),(g)$ and $\mathbf{Q}>100 \mathrm{GeV}(f),(h) . \quad P_{3}$ is now almost unity at $\cos \theta^{*}=-1$, because $\left|\overline{\mathcal{M}}_{-}\left(\theta^{*}\right)\right|=$ $\left|\mathcal{M}_{+}\left(\theta^{*}\right)\right| \approx 0$ at $\theta^{*}=\pi$. As $\cos \theta^{*}$ deviates from $-1, P_{3}$ decreases rapidly and the polarization perpendicular to the helicity axis, $P_{1}$ inside the scattering plane and $P_{2}$ normal to the scattering plane when $\xi \neq 0$ grows, just as in the case of top polarizations shown in the left-hand plots of the figure. Most notably, the magnitude of all three polarization components $P_{1}, P_{2}, P_{3}$ behave very similar as functions of $\cos \theta^{*}$ between the top and the anti-top polarizations for the same $\mathrm{CP}$ phase, whereas their signs are all opposite. As for $P_{2}$, the magnitude becomes the largest for $\mathrm{Q}<100 \mathrm{GeV}$ events at $\mathrm{W}=600 \mathrm{GeV}$, as shown in figure 13(c) for top and (g) for anti-top. As we will explain carefully in the next section, this is a consequence of $\mathrm{CP}$ violation in $\mathrm{CPT}$ invariant theory in the absence of rescattering phase in the amplitudes.

Before we move on studying $t$ and $\bar{t}$ polarization after integration over $\cos \theta^{*}$, we note in figure $13(\mathrm{c})$ and $(\mathrm{g})$ for $\mathrm{Q}<100 \mathrm{GeV}$ at $\mathrm{W}=600 \mathrm{GeV}$, the magnitudes of $P_{2}$ are predicted to be larger for $\xi=0.05 \pi$ (dashed red curve) than those for $\xi=0.1 \pi$ (dash-dotted curve) in the $\cos \theta^{*}>0$ region. This non-linear behavior was not expected for relatively small phase of $|\xi| \leq 0.1 \pi$, and we study the elements of matrix $d \sigma_{\lambda \lambda^{\prime}}$ carefully for ten values of $\xi$ in the range $0<\xi<0.1 \pi$. Shown in the left plot of figure 14 is the $t h j$ production differential cross section, $\sigma_{++}+\sigma_{--}$, with respect to $\cos \theta^{*}$ at $\mathrm{W}=600 \mathrm{GeV}$ for $\mathrm{Q}<100 \mathrm{GeV}$ events. The cross section is smallest at $\xi=0$, and grows with $\xi$ almost linearly in the region $\cos \theta^{*} \gtrsim-0.5$. The cross section near $\cos \theta^{*}=-1$ is dominated by the $W$ exchange amplitudes (with the $A$ factor), and hence does not depend on the $h t t$ coupling. In the middle plot, figure 14(b), we show $\operatorname{Im}(\sigma(+,-))$ v.s. $\cos \theta^{*}$. Its magnitude grows with $\xi$, but it changes sign at around $\cos \theta^{*}=0$ and the growth of the magnitude is very slow at $\cos \theta^{*}>0$. The average polarization $P_{2}$ is obtained as their ratio $-2 \operatorname{Im} \sigma(+,-) /(\sigma(+,+)+\sigma(-,-))$ in eq. (5.5b), which is shown in figure 14(c). In the $\cos \theta^{*}>0$ region, the magnitude grows from $\xi=0$ up to $\xi \simeq 0.05 \pi$, but decreases to the orange curve at $\xi=0.1 \pi$. This study shows that the polarization $P_{2}$ has strong sensitivity to the CP phase $\xi$, whose magnitude can reach $20 \%$ even for $\xi= \pm 0.01 \pi$.

As can be seen from figure 14(a), the differential cross section decreases sharply as $\cos \theta^{*}$ deviates from $\cos \theta^{*}=-1$, and hence the polarization asymmetry integrated over 

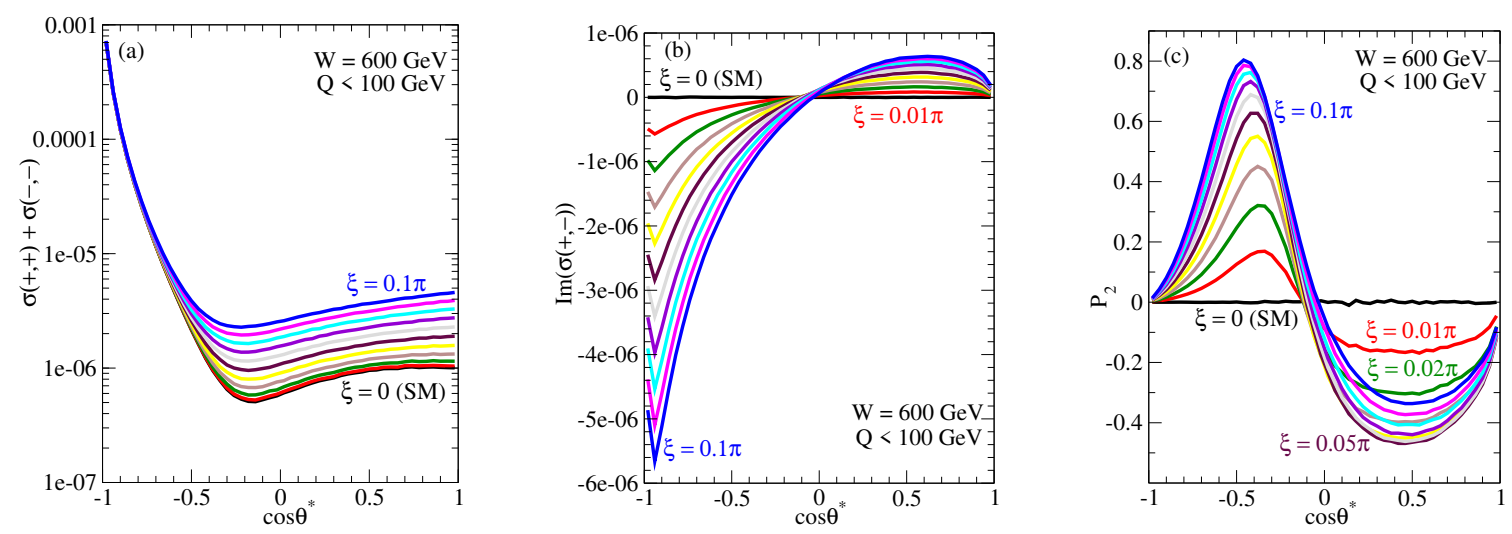

Figure 14. Details of the $\xi$-dependence of the top quark helicity matrix distributions and $P_{2}$ in $p p \rightarrow$ th $j$ at $\mathrm{W}=600 \mathrm{GeV}$ and $\mathrm{Q}<100 \mathrm{GeV}$. (a) $d \sigma / d \cos \theta^{*}=\sigma(+,+)+\sigma(-,-)$ is plotted against $\cos \theta^{*}$, the $t$-quark scattering angle in the $W^{+} b \rightarrow t h$ rest frame. The 11 curves are for $\xi=0$ (SM) and $\xi=n \cdot 0.01 \pi$ with $n=1$ to 10 . (b) Imaginary part of the off-diagonal element of the matrix distribution $d \sigma_{\lambda \lambda^{\prime}}$. (c) The $t$ quark polarization perpendicular to the $W^{+} b \rightarrow$ th scattering plane, $P_{2}=-2 \operatorname{Im} \sigma(+,-) /(\sigma(+,+)+\sigma(-,-))$.

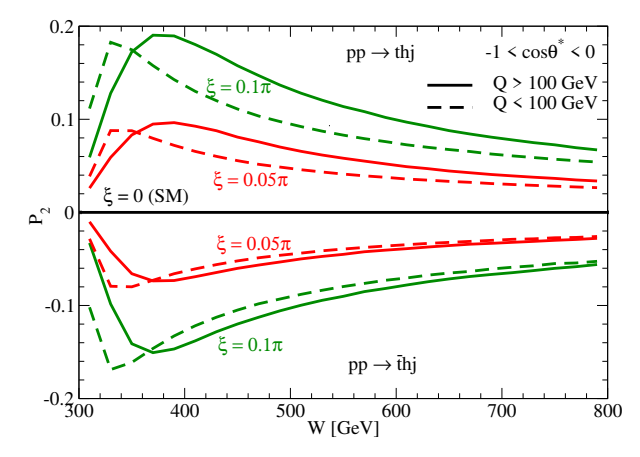

Figure 15. $P_{2}$ v.s. W for $p p \rightarrow t h j$ (a) and $p p \rightarrow \bar{t} h j$ (b) in the region $-1<\cos \theta^{*}<0$. The green curves are for $\xi=0.1 \pi$, while the red curves are for $\xi=0.05 \pi$. The soid curves are for $Q>$ $100 \mathrm{GeV}$, while the dashed curves are for $\mathrm{Q}<100 \mathrm{GeV}$.

$\cos \theta^{*}$ is determined by the sign and magnitude in the $\cos \theta^{*}<0$ region. Shown in figure 15 are the polarization asymmetry $P_{2}$ for top (above zero) and antitop (below zero), for the events with $\cos \theta^{*}<0$, plotted against the $t h(\bar{t} h)$ invariant mass $\mathrm{W}$. The results for $\mathrm{Q}>100 \mathrm{GeV}$ are shown by solid curves, while those for $\mathrm{Q}<100 \mathrm{GeV}$ are shown by dashed curves. The red curves are for $\xi=0.05 \pi$, while green curves are for $\xi=0.1 \pi$. Although the ad-hoc selection cut $\cos \theta^{*}<0$ is not optimal, we can observe the general trend that the magnitude of the polarization asymmetry $P_{2}$ grows with the $\mathrm{CP}$ phase $\xi$, and the sign of $P_{2}$ is positive for $t$, but it is negative for $\bar{t}$, when $\xi>0$.

We may tempt to conclude that the same physics governs the sign of $A_{\phi}$ in figure 11 and that of $P_{2}$ in figure 15, since both asymmetries change sign between thj and $\bar{t} h j$ events. We will study the cause of this similar behaviour in the next section.

Before discussing consequences of CPT invariance in the next section, let us introduce a slightly more complicated top quark polarization asymmetries whose signs also measure the 
sign of $\xi$. We recall that the $t$ polarization perpendicular to the $W^{+} b \rightarrow t h$ scattering plane $P_{2}$ can be expressed as a triple three-vector product (5.6), which is naive T-odd ( $\tilde{\mathrm{T}}$-odd), since it changes the sign when we changes the signs of both the three momentum and spin. In the absence of final state re-scattering phase, $\tilde{\mathrm{T}}$-odd observables measure T-violation, or $\mathrm{CP}$-violation in quantum field theories (QFT). Therefore, we examine pentuple products

$$
\frac{\left(\vec{q} \times \vec{p}_{j}\right) \times\left(\vec{q} \times \vec{p}_{h}\right) \cdot \vec{P}}{\left|\left(\vec{q} \times \vec{p}_{j}\right) \times\left(\vec{q} \times \vec{p}_{h}\right)\right|}, \quad \frac{\left(\vec{p}_{b} \times \vec{p}_{j}\right) \times\left(\vec{p}_{b} \times \vec{p}_{h}\right) \cdot \vec{P}}{\left|\left(\vec{p}_{b} \times \vec{p}_{j}\right) \times\left(\vec{p}_{h} \times \vec{p}_{d}\right)\right|}
$$

which are clearly $\tilde{\mathrm{T}}$-odd polarization asymmetries, whose expectation values should vanish at $\xi=0$ in the tree level. We note that the three-vector $\left(\vec{q} \times \overrightarrow{p_{j}}\right) \times\left(\vec{q} \times \vec{p}_{h}\right)$ points toward the direction of $\vec{q}$, while its sign changes when the azimuthal angle between the $W^{+}$emission plane and the $W^{+} b \rightarrow t h$ scattering plane changes sign, between $-\pi<\phi<0$ and $0<\phi<\pi$. Likewise, $\left(\vec{p}_{b} \times \vec{p}_{j}\right) \times\left(\vec{p}_{b} \times \vec{p}_{h}\right)$ points either along or opposite of $\vec{p}_{b}$ direction, depending on the same azimuthal angle between the two planes, because the $W^{+}$momentum $\vec{q}$ and the $b$ momentum $\vec{p}_{b}$ are back to back in the frames which define the emission and the scattering planes, see figure 3. In the top quark rest frame, the two three-vectors, $\vec{q}$ and $\vec{p}_{b}$ span the scattering plane, which is chosen as the $x$ - $z$ plane in our analysis. Therefore, if we define the azimuthal asymmetry of the top quark polarization vector as

$$
P_{k}^{A}=P_{k}(\phi>0)-P_{k}(\phi<0),
$$

where $P_{k}(\phi>0)$ and $P_{k}(\phi<0)$ denotes, respectively, the top quark polarization of events with $\phi>0$ and $\phi<0, P_{1}^{A}$ and $P_{3}^{A}$ are $\tilde{\mathrm{T}}$-odd. ${ }^{4}$ This is because the $x$ - and $z$-axis vectors are linear combination of $\vec{q}$ and $\vec{p}_{b}$ in the $t$-rest frame.

We show in figure 16 all three polarization asymmetries, $P_{k}^{A}$ for $k=1,2,3$, for $p p \rightarrow t h j$ events in the left two panels $(a),(b)$, and for $p p \rightarrow \bar{t} h j$ in the right panels. The upper plots in figure $16(a),(c)$ are for $\mathrm{W}=400 \mathrm{GeV}$, while the bottom plots $(b),(d)$ are for $\mathrm{W}=600 \mathrm{GeV}$, both for $\mathrm{Q}>100 \mathrm{GeV}$. As expected, $P_{1}^{A}=P_{3}^{A}=0$ for the $\mathrm{SM}(\xi=0)$. We find that $P_{3}^{A}>0$ for $\xi=0.05 \pi$ (dashed curves) and $0.1 \pi$ (dash-dotted curves) in all the regions of $\cos \theta^{*}, \mathrm{~W}$, and $\mathrm{Q}$ that we study, including the four cases shown in figure 16 . This follows our observation that $P_{3}$ is large and opposite in sign between $t$ and $\bar{t}$, see figure 13 , and that azimuthal angle asymmetry is also opposite in sign, see figure 11. The magnitude of $P_{1}^{A}$ is small near $\cos \theta^{*}=-1$ where the cross section is large.

\section{T-odd v.s. CPV asymmetry}

As explained in the previous sections, the asymmetries $A_{\phi}, P_{2}, P_{1}^{A}$ and $P_{3}^{A}$, whose signs measure the sign of the $\mathrm{CP}$ violating phase $\xi$ are all so-called T-odd asymmetries. We found in section 4 that the asymmetry $A_{\phi}$ has opposite sign between the $p p \rightarrow$ thj events

\footnotetext{
${ }^{4}$ The azimuthal asymmetry of the top quark polarization is not normalized to the sum $P_{k}(\phi>0)+P_{k}(\phi<$ 0 ), because the sum cannot be interpretted as the polarization $P_{k}$ of the whole events. This is because $P_{k}(\phi>0)$ is normalized to the number of events in the $\phi>0$ region, whereas $P_{k}(\phi<0)$ is normalized to the number of events in the $\phi<0$ region. Only when the number of events are the same, i.e. $A_{\phi}=0$, the sum becomes $P_{k}$.
} 

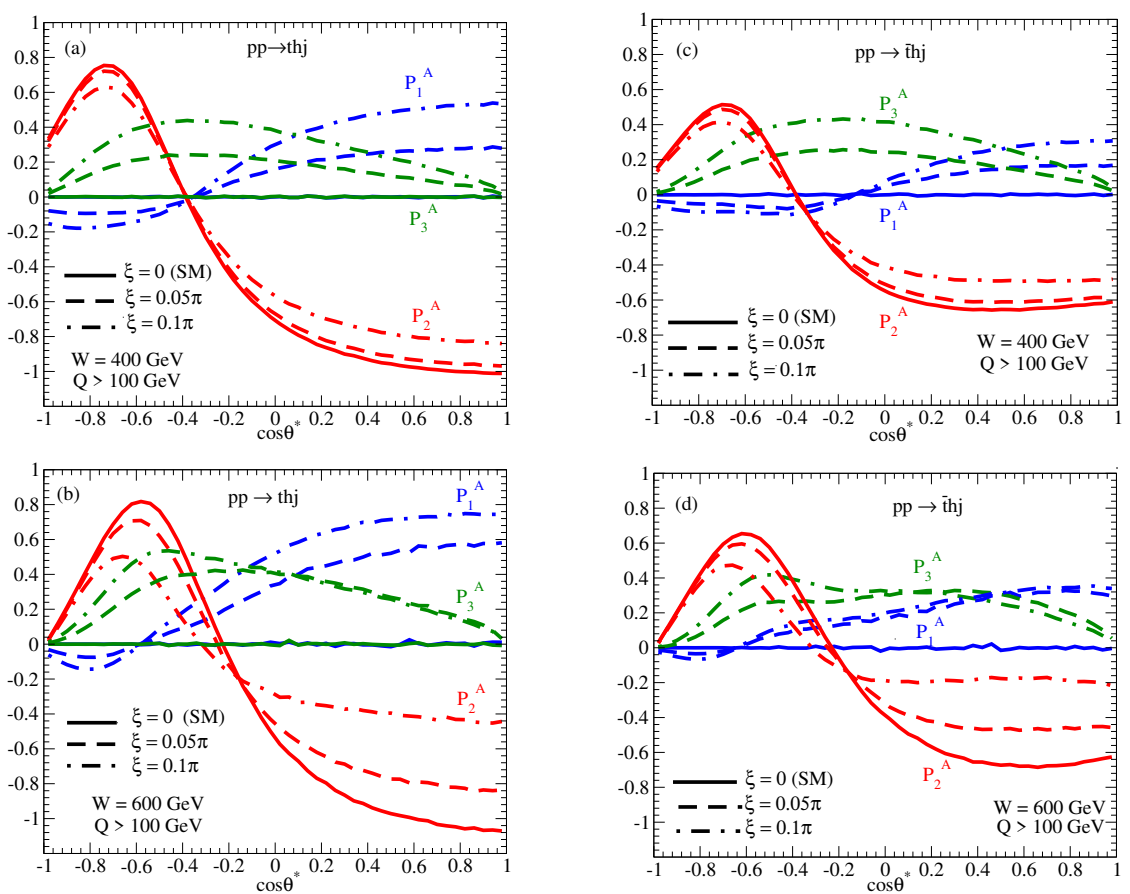

Figure 16. $P_{1,2,3}^{A}$ v.s. $\cos \theta^{*}$ for $\mathrm{Q}>100 \mathrm{GeV}$ events at -1 for $\mathrm{W}=400(a)$, (c), and $600 \mathrm{GeV}$ $(b),(d)$. The left-hand plots $(a),(b)$ are for $p p \rightarrow t h j$ events, while the right-hand plots $(c),(d)$ are for $p p \rightarrow \bar{t} h j$ events. The solid, dashed, and dash-dotted curves are for $\xi=0$ (SM), $\xi=0.05 \pi$ and $\xi=0.1 \pi$, respectively. $P_{1}^{A}=P_{3}^{A}=0$ for $\xi=0$ (SM).

and $p p \rightarrow \bar{t} h j$ events, and we found in section 5 the polarization asymmetry $P_{2}$ has the opposite sign between the thj and $\bar{t} h j$ events. In this section, we study consequences of the invariance under the discrete unitary transformations $\tilde{\mathrm{T}}$ and $\mathrm{CP}$, and CP $\tilde{\mathrm{T}}$.

We adopt the symbol $\tilde{\mathrm{T}}$ for the unitary transformation under which all the three momenta $\vec{p}$ and the spin vectors $\vec{s}$ reverse their sign, in order to distinguish it from the time reversal transformation $\mathrm{T}$, which reverses the sign of the time direction, and hence is anti-unitary. In the absence of the final state interaction phases of the amplitudes, $\tilde{\mathrm{T}}$-odd asymmetries are proportional to $\mathrm{T}$ violation, or equivalently $\mathrm{CP}$ violation in QFT.

Figure 17 illustrates the $\tilde{\mathrm{T}}$ and $\mathrm{CP}$ transformations of the subprocess $u b \rightarrow d t h$, whose three momenta are the same as those in figure 3, or eqs. (2.13) and (2.11). We add the helicities of external massless quarks $(u, d, b)$ and also along the $W^{+}$momentum direction, where the $\lambda=-1$ state is chosen for illustration. The top polarization, or its decay charged lepton momentum, is normal to the scattering plane along the positive $y$-axis. Under $\tilde{T}$ transformation, all the three momenta and spin polarizations change sign, as shown in (b), which can be viewed as $(d)$ by making the 180 degree rotation about the $y$-axis. Comparing $(a)$ and $(d)$, we find that the initial state remains the same while in the final state

$$
\phi \rightarrow-\phi \quad \text { and } \quad P_{2} \rightarrow-P_{2}
$$

under $\tilde{\mathrm{T}}$ transformation. Therefore the observation of $\tilde{\mathrm{T}}$-odd asymmetries such as

$$
A_{\phi} \neq 0 \quad \text { or } \quad P_{2} \neq 0
$$




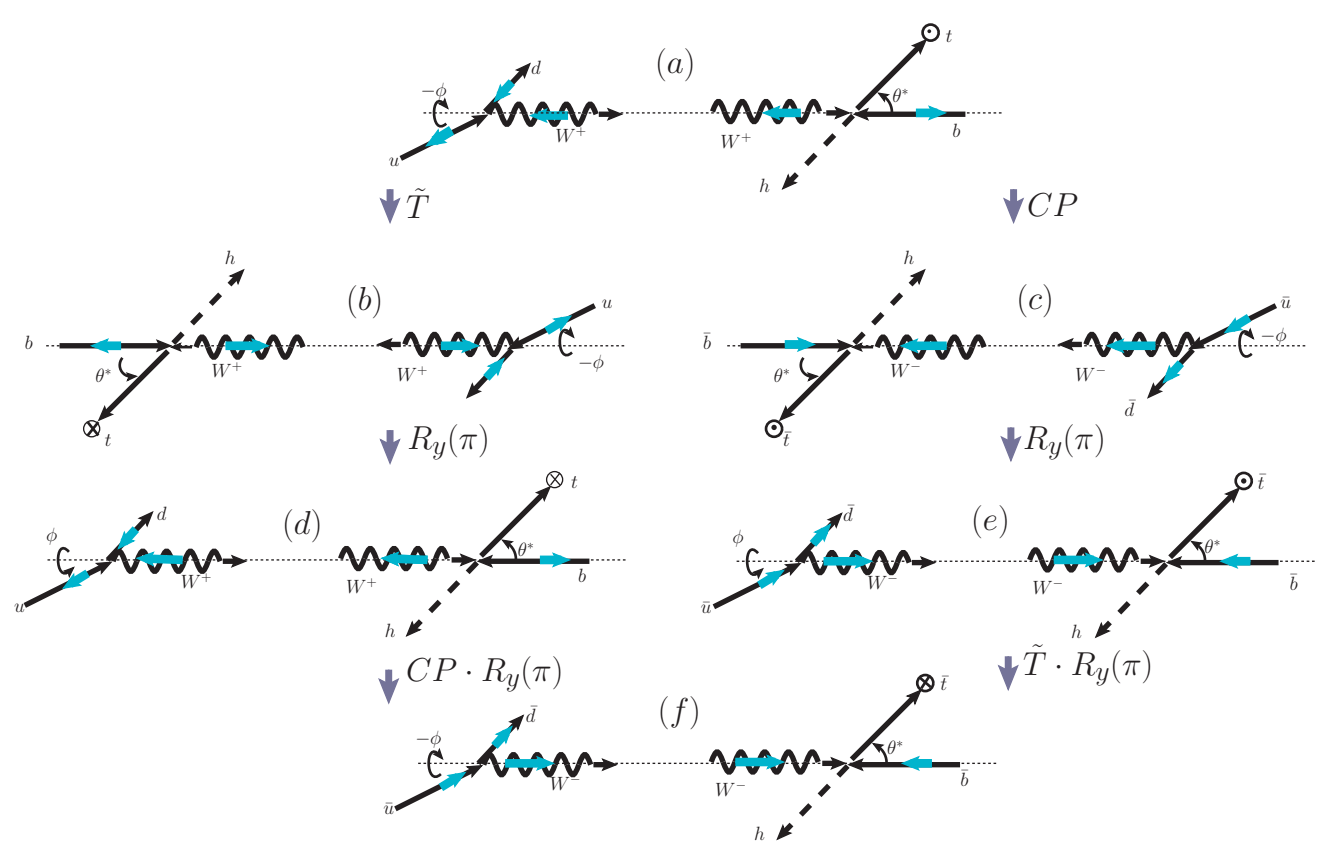

Figure 17. Illustration of $\tilde{\mathrm{T}}$ and $\mathrm{CP}$ transformations of the process $u b \rightarrow d t h$. (a) shows the three momenta and polarization of $u \rightarrow d W^{+}$and $W^{+} b \rightarrow t h$ as parametrized in figure 3 . The $W^{+} b \rightarrow t h$ scattering is in the $z$-x plane, $u$ and $d$ three momenta have negative $y$-components, and the top polarization is along the positive $y$-axis. $(b)$ is obtained from $(a)$ by $\tilde{\mathrm{T}}$ transformation, while $(c)$ is obtained from $(a)$ by CP transformation. $(d)$ and $(e)$ are obtained, respectively, from $(b)$ and $(c)$ by a 180 degree rotation about the $y$-axis. $(f)$ is obtained from $(d)$ by $\mathrm{CP}$, or from $(e)$ by $\tilde{\mathrm{T}}$ transformation, together with the 180 degrees rotation about the $y$-axis.

implies either T-violation or the presence of an absorptive phase of the scattering amplitudes or both [40, 41].

Likewise, the configuration $(c)$ or $(e)$ after the $R_{y}(\pi)$ rotation, is obtained by CP transformation from the configuration $(a)$. All the particles are transformed to anti-particles and their helicities and three momenta are reversed. If we define the asymmetries $\bar{A}_{\phi}$ and $\bar{P}_{2}$ for the process $\bar{p} \bar{p} \rightarrow \bar{t} h j$, then CP-invariance between $(a)$ and $(e)$ implies

$$
\bar{A}_{\phi}=-A_{\phi} \quad \text { and } \quad \bar{P}_{2}=P_{2} .
$$

Violation of the above identities hence gives CP-violation.

Finally, the configuration $(f)$ in figure 17 is obtained from $(d)$ by applying $\mathrm{CP}$, or from (e) by applying $\tilde{\mathrm{T}}$, together with the rotation $R_{y}(\pi)$. In short, $(f)$ is obtained from our original configuration $(a)$ by $\mathrm{CP} \tilde{\mathrm{T}}$ transformation [42]. By comparing $(a)$ and $(f), \mathrm{CP} \tilde{\mathrm{T}}$ invariance, or the absence of the absorptive phase in QFT amplitudes should give

$$
\bar{A}_{\phi}=A_{\phi} \quad \text { and } \quad \bar{P}_{2}=-P_{2}
$$

As an illustration of how absorptive phases of the amplitudes in $\mathrm{T}$ or $\mathrm{CP}$ invariant theory contribute to $\tilde{T}$-odd asymmetries, we examine the impacts of the top-quark width in the $s$-channel propagator $D_{t}\left(P_{t h}\right)$ in eq. (2.4), or in the $B$ factor of eq. (2.25b). The 



Figure 18. T- -odd asymmetries $A_{\phi}$ (a) and $P_{2}$ (b) due to the top quark width $\Gamma_{t}$ in $\mathrm{CP}$ invariant theory $(\xi=0)$ in $p p \rightarrow t h j$ (solid lines) and $\bar{p} \bar{p} \rightarrow \bar{t} h j$ (dashed lines) at $\mathrm{W}=600 \mathrm{GeV}$ for events with $\mathrm{Q}<100 \mathrm{GeV}$. Three values of $\Gamma_{t}$ are shown: $\Gamma_{t}=0$ (blue), $\Gamma_{t}=1.35 \mathrm{GeV}$ (red), and ten times the SM value $\Gamma_{t}=13.5 \mathrm{GeV}$ (green).

width of Breit-Wigner propagator gives absorptive parts to our amplitudes, and since the top quark width appears only in the amplitudes with $h t t$ coupling, it can give rise to $\tilde{\mathrm{T}}$-odd asymmetries, $A_{\phi}$ and $P_{2}$. We show in figure 18 the asymmetries $A_{\phi}(a)$ and $P_{2}(b)$ in the CPinvariant $\mathrm{SM}(\xi=0)$ for $\Gamma_{t}=0$ (blue), $\Gamma_{t}=1.35 \mathrm{GeV}$ (red), the SM value, and for 10 times the SM width $\Gamma_{t}=13.5 \mathrm{GeV}$ (green). We find that the asymmetries are both zero when $\Gamma_{t}=0$ as expected. Furthermore, we confirm the relations (6.3) between the asymmetries of $p p \rightarrow t h j$ events, $A_{\phi}$ and $P_{2}$, and those of $\bar{p} \bar{p} \rightarrow \bar{t} h j$ events, $\bar{A}_{\phi}$ and $\bar{P}_{2}$, respectively. This is a consequence of $\mathrm{CP}$ invariance, as can be viewed from the illustration by comparing the configurations $(a)$ and $(e)$. If $\mathrm{CP}$ is conserved, the amplitudes for the configuration $(e)$ should have the same magnitude with those of the original configuration $(a)$. The azimuthal angle between the $W$ emission plane and the scattering plane is reversed, whereas the $\bar{t}$ spin polarization should be the same as the $t$ spin polarization.

It is worth noting here that instead of top and anti-top spin polarization vector, $P_{2}$ and $\bar{P}_{2}$, if we use the decay charged lepton momentum normal to the scattering plane in the $t$ or $\bar{t}$ rest frame, we find

$$
\left\langle p_{y}^{l^{-}}\right\rangle=-\left\langle p_{y}^{l^{+}}\right\rangle
$$

as a consequence of $\bar{P}_{2}=P_{2}$ (6.3) in CP invariant theory. Here, we assume that the $t$ and $\bar{t}$ decay angular distributions follow the SM, where the charged leptons are emitted preferably along the $t$ spin polarization direction, whereas they are emitted in the opposite of the $\bar{t}$ spin polarization direction. This is simply because only the right-handed $l^{+}$and the left-handed $l^{-}$are emitted from $t$ and $\bar{t}$ decays, respectively, in the SM. The above spinmomentum correlation is $\mathrm{CP}$ invariant, and hence the identity (6.5) is also a consequence of CP invariance.

In figure 19, we show comparisons of the asymmetries between $p p \rightarrow t h j$ and $\bar{p} \bar{p} \rightarrow \bar{t} h j$ events for $\mathrm{CP}$ violating theory $(\xi \neq 0)$ in the approximation of no absorptive parts in the amplitudes, i.e., we set $\Gamma_{t}=0$. We confirm the relations (6.4) for the same value of $\xi$, as a consequence of CP $\tilde{\mathrm{T}}$ invariance. The relations between the asymmetries in $p p \rightarrow t h j$ and $\bar{p} \bar{p} \rightarrow \bar{t} h j$ are opposite between figure 18 and figure 19, as expected from eqs. (6.3) and (6.4). 

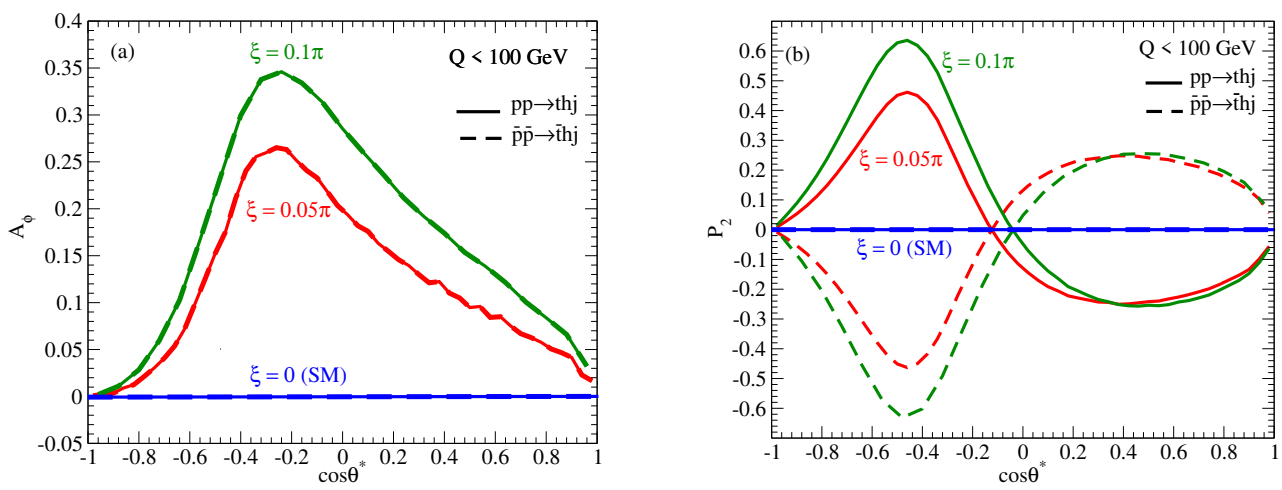

Figure 19. The azimuthal angle asymmetry $A_{\phi}(a)$ and the top (anti-top) polarization asymmetry $P_{2}(b)$ in $p p \rightarrow t h j$ events (solid curves) and $\bar{p} \bar{p} \rightarrow \bar{t} h j$ events (dashed curve) for $\xi=0$ (SM, blue curve), and $\xi=0.05 \pi$ (red curve).

All the above relations between $p p$ and $\bar{p} \bar{p}$ may seem to be just formal rules since we will not have a $\bar{p} \bar{p}$ collider with the LHC energy and luminosity. However, we find the above rules useful in testing our amplitudes, especially in fixing the relative sign between the two helicity amplitudes which determines the top and anti-top spin polarization directions away from their helicity axis. Furthermore, we find that it is possible to disentangle T-odd effects coming from the SM re-scattering effects (that give rise to the absorptive amplitudes) from $\mathrm{CP}$ violating new physics effects in $p p$ collisions at the LHC by measuring the polarization asymmetry $P_{2}$ of $t$ and $\bar{t}$ precisely.

Let us examine figure 15 again, where we show $P_{2}$ for $t h j$ and $\bar{t} h j$ events at the LHC as a function of $\mathrm{W}$, the $t h$ or $\bar{t} h$ invariant mass. The polarization asymmetry $P_{2}$ have opposite sign between $t$ and $\bar{t}$. More quantitatively, we note that the magnitudes of the asymmetry is almost the same for small $\mathrm{Q}$ events $(\mathrm{Q}<100 \mathrm{GeV})$ at large $\mathrm{W}(\mathrm{W} \gtrsim 600 \mathrm{GeV})$. This is a consequence of CP $\tilde{T}$ invariance of our tree-level amplitudes with $\Gamma_{t}=0$, because at small $\mathrm{Q}$ and large $\mathrm{W}$, the events are dominated by the contributions of the longitudinally polarized $W$ bosons; see figure 7 (a) and (b). Therefore, in this region of the phase space, we can regard the single top or anti-top plus Higgs production processes as

$$
\begin{aligned}
& W^{+}(\lambda=0)+b \rightarrow t+h \\
& W^{-}(\lambda=0)+\bar{b} \rightarrow \bar{t}+h
\end{aligned}
$$

which are $\mathrm{CP}$ conjugates of each other. Their amplitudes are given in eqs. (2.22c) and (2.35), and we can obtain the polatization asymmetries directly from these amplitudes, which are independent of parton distribution functions in $p p$ collisions.

Because the absorptive amplitudes contribute to the polarization asymmetry $P_{2}$ with the same sign as shown in figure 18, we can further tell that the difference,

$$
P_{2}(t h j \text { events })-P_{2}(\bar{t} h j \text { events })
$$

measures CP violation, whereas the sum

$$
P_{2}(t h j \text { events })+P_{2}(\bar{t} h j \text { events })
$$


measures the CPT̃-odd effects from the absorptive amplitudes in the region of small $\mathrm{Q}$ and large W. We find in the SM the leading contributions for the absorptive amplitudes appear at one-loop level in QCD and in the electroweak theory [43]. The top quark width that we adopted in this section for illustration is a part of the electroweak corrections.

The sign of the polarization asymmetry $P_{2}$ remains the same and the magnitudes are larger at smaller $\mathrm{W}$ and large $\mathrm{Q}$. This can be understood qualitatively also from figure 7 , where the sub-dominant contributions are

$$
\begin{aligned}
& W^{+}(\lambda=-1)+b \rightarrow t+h \\
& W^{-}(\lambda=+1)+\bar{b} \rightarrow \bar{t}+h
\end{aligned}
$$

at small $\mathrm{W}(\mathrm{W} \lesssim 500 \mathrm{GeV})$ especially at large $\mathrm{Q}(\mathrm{Q}>100 \mathrm{GeV})$. The above subprocesses are again $\mathrm{CP}$-conjugate to each other, and hence follow the rule (6.4) from $\mathrm{CP} \tilde{\mathrm{T}}$ invariance.

\section{Summary and discussion}

We studied associated production of single top (or anti-top) and the Higgs boson via $t$ channel $W$ exchange at the LHC. We obtained analytically the helicity amplitudes for all the tree-level subprocesses with massless $b$ (or $\bar{b}$ ) quark PDF in the proton, and studied consequences of possible $\mathrm{CP}$ violation in the Higgs Yukawa coupling to the top quark. By choosing the momentum direction of the $W^{ \pm}$exchanged in the $t$-channel, the helicity amplitudes are factorized into the $W^{ \pm}$emission amplitudes from light quarks or antiquarks, and the $W^{+} b \rightarrow t h$ or $W^{-} \bar{b} \rightarrow \bar{t} h$ production amplitudes. We find that the amplitudes for the right-handed top quark and those of the left-handed anti-top quark are sensitive to the sign of the $\mathrm{CP}$ violating phase $\xi$ in the effective Yukawa interaction Lagrangian of eq. (1.1). This is because the right-handed top quark is produced by the $t_{R}^{\dagger} t_{L}$ operator with the $e^{-i \xi}$ phase without chirality suppression, whereas the contribution of the $t_{L}^{\dagger} t_{R}$ operator with the $e^{i \xi}$ phase is doubly suppressed. For the anti-top production, the role of the two operators are reversed. On the other hand, the other amplitudes for the left-handed top and the right-handed anti-top productions are almost proportional to $e^{i \xi}+e^{-i \xi}=2 \cos \xi$ because both terms in the Lagrangian contribute with one chirality suppression, either in the top quark propagator or from the helicity-chirality mismatch in the wave function, $\delta^{\prime}$ and $\delta$ in eq. (2.26), respectively.

We studied mainly the azimuthal angle asymmetry $A_{\phi}$ between the $W^{ \pm}$emission plane and the $W^{+} b \rightarrow t h$ or $W^{-} \bar{b} \rightarrow \bar{t} h$ production plane, and the $t$ or $\bar{t}$ spin polarization normal to the scattering plane, $P_{2}$, as observables which are sensitive to the sign of the CP phase $\xi$. The asymmetry $A_{\phi}$ arises from the interference between the amplitudes with longitudinal and transversely polarized $W^{ \pm}$contributions, and hence is significant when the exchanged momentum transfer $\mathrm{Q}$ is relatively large and the $t h$ or $\bar{t} h$ invariant mass $\mathrm{W}$ is not too large, where both of the interfering amplitudes are significant. The magnitude of the asymmetry can be enhanced by selecting the chirality favored top or anti-top quark helicity, e.g. by selecting those events with charged lepton momentum along the top or anti-top momentum direction in the $t h$ or $\bar{t} h$ rest frame; see figure 11. 
On the other hand, the polarization asymmetry $P_{2}$ is obtained as the interference between the two helicity amplitudes of $t$ or $\bar{t}$. We find that the amplitudes are dominated by the collision of longitudinally polarized $W^{ \pm}$and $b$ or $\bar{b}$ when the momentum transfer $\mathrm{Q}$ is small and the invariant mass $\mathrm{W}$ of the $t h$ or $\bar{t} h$ system is large. Therefore in such kinematical configuration, the asymmetry $P_{2}$ of the top and the anti-top can be regarded as the direct test of $\mathrm{CP}$ violation between the CP-conjugate processes, $W^{+}(\lambda=0)+b \rightarrow t+h$ and $W^{-}(\lambda=0)+\bar{b} \rightarrow \bar{t}+h$. Because of the dominance of the longitudinally polarized $W^{ \pm}$ exchange amplitudes, all the differences in the quark and anti-quark PDF's of the colliding protons drop out in the polarization asymmetry.

All the analytic and numerical results presented in this report are done strictly in the tree-level, in order to clarify the symmetry properties of observable asymmetries that are sensitive to the sign of the CP violating phase $\xi_{h t t}$. In order to show their observability at the HL-LHC with its $3 \mathrm{ab}^{-1}$ of integrated luminosity, we should perform the following studies.

Most importantly, we should identify the top and the Higgs decay modes which can be used to measure the asymmetries, since we may have different radiative corrections and background contributions for each set of the decay modes. We expect that semi-leptonic decays of $t$ and $\bar{t}$ when the Higgs decays into modes without missing energy are favorable because the lepton charge identify $t$ vs. $\bar{t}$, and the charged lepton decay anglular distribution measures the $t$ and $\bar{t}$ polarization with maximum sensitivity. Hadronically decaying $t$ and $\bar{t}$ events can have sensitivity to the asymmetries, because their decay density matrix polarimeter introduced in ref. [37] retains strong sensitivity to the $t$ and $\bar{t}$ polarizations, and also because the $\mathrm{CP}$ asymmetry of the polarizations, $P_{2}(\bar{t} h j) \approx-P_{2}(t h j)$ in figure 15 tells that the observable asymmetries in the decay distributions are the same between $t$ and $\bar{t}$ events even if we cannot distinguish between them. Although the direct test of CP violation cannot be made in the hadronic decay modes, the sensitivity to the sign and the magnitude of the $\mathrm{CP}$ violating phase $\xi$ can be improved by assuming the SM radiative contribution to the asymmetries [43].

We believe that the associated production of the Higgs boson and single $t$ or $\bar{t}$ via $t$-channel $W^{ \pm}$exchange at the LHC can be an ideal testing ground of the top quark Yukawa coupling, because the amplitudes with the htt Yukawa coupling and those of the $h W W$ coupling interfere strongly. We studied the sensitivity of the process to possible CP violation in the Yukawa coupling. We anticipate that our studies based on the analytic form of the helicity amplitudes will be useful in the test of various scenarios of physics beyond the SM. 


\section{A Helicity amplitudes and the top spin orientation}

The helicity amplitudes $M_{+}$and $M_{-}$in the single top plus Higgs production processes via $t$-channel $W$ exchange are pure complex numbers when all the other quark masses are set to be zero, because all their helicities are fixed by the SM $V-A$ interactions and because the Higgs boson has no spin. The produced top quark is hence a pure quantum state

$$
|t\rangle=\frac{M_{+}}{\sqrt{\left|M_{+}\right|^{2}+\left|M_{-}\right|^{2}}}\left(\begin{array}{l}
1 \\
0
\end{array}\right)+\frac{M_{-}}{\sqrt{\left|M_{+}\right|^{2}+\left|M_{-}\right|^{2}}}\left(\begin{array}{l}
0 \\
1
\end{array}\right)
$$

in the top rest frame. Its spin is polarized in the positive $z$-direction, $J_{z}=\frac{1}{2}$, if $M_{+} \neq 0$ and $M_{-}=0$, where the $z$-axis is along the top quark momentum direction where its helicity is defined. If $M_{+}=0$ but $M_{-} \neq 0$, the top quark has $J_{z}=-\frac{1}{2}$. In general, the spin polarization of the top quark can have an arbitrary spatial orientation

$$
\vec{J}=\frac{1}{2} \vec{P}=\frac{1}{2}(\sin \theta \cos \phi, \sin \theta \sin \phi, \cos \theta),
$$

where $\theta$ and $\phi$ are polar and azimuthal angles about the $z$-axis. The corresponding top state can be obtained from the $\vec{P}=(0,0,1)$ state by two rotations,

$$
\begin{aligned}
|t, \vec{P}(\theta, \phi)\rangle & =R_{z}(\phi) R_{y}(\theta)\left(\begin{array}{l}
1 \\
0
\end{array}\right) \\
& =\left(\begin{array}{cc}
e^{-i \frac{\phi}{2}} & 0 \\
0 & e^{i \frac{\phi}{2}}
\end{array}\right)\left(\begin{array}{cc}
\cos \frac{\theta}{2}-\sin \frac{\theta}{2} \\
\sin \frac{\theta}{2} & \cos \frac{\theta}{2}
\end{array}\right)\left(\begin{array}{l}
1 \\
0
\end{array}\right) \\
& =e^{-i \frac{\phi}{2}} \cos \frac{\theta}{2}\left(\begin{array}{l}
1 \\
0
\end{array}\right)+e^{i \frac{\phi}{2}} \sin \frac{\theta}{2}\left(\begin{array}{l}
0 \\
1
\end{array}\right)
\end{aligned}
$$

By comparing (A.1) and (A.3), we find

$$
\frac{M_{-}}{M_{+}}=e^{i \phi} \tan \frac{\theta}{2}
$$

or

$$
\theta=2 \tan ^{-1}\left|\frac{M-}{M_{+}}\right|, \quad \phi=\arg \left(\frac{M_{-}}{M_{+}}\right) .
$$

For the mixed states, it is useful to introduce the density matrix

$$
\rho_{\sigma \sigma^{\prime}}=\frac{1}{\sum\left(\left|M_{+}\right|^{2}+\left|M_{-}\right|^{2}\right)}\left(\begin{array}{cc}
\sum\left|M_{+}\right|^{2} & \sum M_{+} M_{-}^{*} \\
\sum M_{+}^{*} M_{-} & \sum\left|M_{-}\right|^{2}
\end{array}\right)
$$

where the summation is over all the processes and kinematical configurations that contribute to the top quark which we observe. Because the matrix is Hermitian and has trace 1 , we can parametrize it as

$$
\rho=\frac{1}{2}(1+\vec{P} \cdot \vec{\sigma})
$$


by using the $\vec{\sigma}$ matrices. We find

$$
P_{1}=\frac{2 \operatorname{Re} \sum\left(M_{+} M_{-}^{*}\right)}{\sum\left(\left|M_{+}\right|^{2}+\left|M_{-}\right|^{2}\right)}, \quad P_{2}=\frac{-2 \operatorname{Im} \sum\left(M_{+} M_{-}^{*}\right)}{\sum\left(\left|M_{+}\right|^{2}+\left|M_{-}\right|^{2}\right)}, \quad P_{3}=\frac{\sum\left(\left|M_{+}\right|^{2}-\left|M_{-}\right|^{2}\right)}{\sum\left(\left|M_{+}\right|^{2}+\left|M_{-}\right|^{2}\right)},
$$

which for the pure state (A.1) gives (A.2).

In general, we can parametrize the density matrix (A.7) as

$$
\rho=\frac{1-|\vec{P}|}{2}+\frac{|\vec{P}|}{2}\left(1+\frac{\overrightarrow{P \cdot \vec{\sigma}}}{|\vec{P}|}\right),
$$

which is a sum of unpolarized top quark with the probability $1-|\vec{P}|$, and the fully polarized top quark with its spin polarization orientation along

$$
\vec{P}=|\vec{P}|(\sin \theta \cos \phi, \cos \theta \sin \phi, \cos \theta)
$$

with the probability $|\vec{P}|$. We find it convenient to show the general polarization vector $\vec{P}$ (A.10) by using an arrow of length $|\vec{P}|$ in the polar coordinate defined as

$$
-\pi<\theta \leq \pi, \quad \frac{-\pi}{2}<\phi \leq \frac{\pi}{2} .
$$

When the imaginary part of $M_{-} / M_{+}$is small, we tend to have small $|\phi|$, and with the above definition we can show $\phi>0$ and $\phi<0$ as pointing up and down in the $z$-x plane [32].

\section{B Polarized $t$ and $\bar{t}$ decay distributions}

The general mixed state of $t$ and $\bar{t}$ in a given kinematical configuration is described by the polarization density matrix (A.7) with the polarization vector, $\vec{P}=\left(P_{1}, P_{2}, P_{3}\right)$ with $|\vec{P}|<1$. In this appendix we give $t$ and $\bar{t}$ decay angular distributions for both semi-leptonic and hadronic decay modes.

The decay density matrix for semi-leptonic decay modes is very simple because it depends only on the charged lepton polar and azimuthal angles [28, 37, 39]

$$
\begin{aligned}
& d \rho\left(t \rightarrow b \ell^{+} \nu\right)=B(t \rightarrow b \ell \nu)\left(\begin{array}{cc}
1+\cos \bar{\theta}^{*} & \sin \bar{\theta}^{*} e^{i \bar{\phi}^{*}} \\
\sin \bar{\theta}^{*} e^{-i \bar{\phi}^{*}} & 1-\cos \bar{\theta}^{*}
\end{array}\right) \frac{d \cos \bar{\theta}^{*} d \bar{\phi}^{*}}{4 \pi} \\
& d \rho\left(\bar{t} \rightarrow \bar{b} \ell^{-} \bar{\nu}\right)=B(t \rightarrow b \ell \nu)\left(\begin{array}{cc}
1-\cos \theta^{*} \sin \theta^{*} e^{-i \phi^{*}} \\
\sin \theta^{*} e^{i \bar{\phi}^{*}} & 1+\cos \theta^{*}
\end{array}\right) \frac{d \cos \theta^{*} d \phi^{*}}{4 \pi}
\end{aligned}
$$

where the $\ell^{ \pm}$four momenta in the $t$ and $\bar{t}$ rest frame, respectively, are parametrized as

$$
\begin{aligned}
& p_{\ell^{+}}^{\mu}=\frac{m_{t}}{2} \bar{x}\left(1, \sin \bar{\theta}^{*} \cos \bar{\phi}^{*}, \sin \bar{\theta}^{*} \sin \bar{\phi}^{*}, \cos \bar{\theta}^{*}\right), \\
& p_{\ell^{-}}^{\mu}=\frac{m_{t}}{2} x\left(1, \sin \theta^{*} \cos \phi^{*}, \sin \theta^{*} \sin \phi^{*}, \cos \theta^{*}\right) .
\end{aligned}
$$


The $z$-axis is along the $t$ or $\bar{t}$ helicity axis and the $y$-axis is along the normal to the scattering plane, $\vec{q} \times \vec{p}_{t}$ or $\vec{q} \times \vec{p}_{\vec{t}}$, respectively, where the helicity amplitudes are calculated. ${ }^{5}$ The decay angular distributions are then

$$
\begin{aligned}
d \Gamma(t(\vec{P}) \rightarrow b \bar{\ell} \nu) & =\sum_{\sigma} \sum_{\sigma^{\prime}} \rho_{\sigma \sigma^{\prime}}^{t}(\vec{P}) d \rho(t \rightarrow b \bar{\ell} \nu)_{\sigma \sigma^{\prime}} \\
& =B(t \rightarrow b \bar{\ell} \nu)\left\{1+P_{1} \sin \bar{\theta}^{*} \cos \bar{\phi}^{*}+P_{2} \sin \bar{\theta}^{*} \sin \bar{\phi}^{*}+P_{3} \cos \bar{\theta}^{*}\right\} \frac{d \cos \bar{\theta}^{*} d \bar{\phi}^{*}}{4 \pi}, \\
d \Gamma(\bar{t}(\vec{P}) \rightarrow \bar{b} \ell \bar{\nu}) & =\sum_{\sigma} \sum_{\sigma^{\prime}} \rho_{\sigma \sigma^{\prime}}^{\bar{t}}(\vec{P}) d \rho(\bar{t} \rightarrow \bar{b} \ell \bar{\nu})_{\sigma \sigma^{\prime}} \\
& =B(t \rightarrow b \bar{\ell} \nu)\left\{1-P_{1} \sin \theta^{*} \cos \phi^{*}-P_{2} \sin \theta^{*} \sin \phi^{*}-P_{3} \cos \bar{\theta}^{*}\right\} \frac{d \cos \theta^{*} d \phi^{*}}{4 \pi} .
\end{aligned}
$$

The $t$ and $\bar{t}$ decay density matrix distributions for hadronic decay modes are slightly more complicated because it is difficult to identify the down-type quark jet from the uptype quark jet in the $W^{+} \rightarrow \bar{d} u(\bar{s} c)$ and $W^{-} \rightarrow d \bar{u}(s \bar{c})$ dijet system. By assuming that the $b$ and $\bar{b}$ jet can be identified uniquely,

$$
\begin{aligned}
& p_{b}^{\mu}=\frac{m_{t}}{2} x_{b}\left(1, \sin \theta_{b}^{*} \cos \phi_{b}^{*}, \sin \theta_{b}^{*} \sin \phi_{b}^{*}, \cos \theta_{b}^{*}\right), \\
& p_{\bar{b}}^{\mu}=\frac{m_{t}}{2} x_{\bar{b}}\left(1, \sin \theta_{\bar{b}}^{*} \cos \phi_{\bar{b}}^{*}, \sin \theta_{\bar{b}}^{*} \sin \phi_{\bar{b}}^{*}, \cos \theta_{\bar{b}}^{*}\right),
\end{aligned}
$$

in the $t$ and $\bar{t}$ rest frame, respectively, with $x_{b}=x_{\bar{b}}=1-m_{W}^{2} / m_{t}^{2}$ in the narrow $W$ width approximation, the $\bar{d}$ and $u$ four momenta are parametrized in the $W^{+} \rightarrow d \bar{u}$ rest frame as

$$
\begin{aligned}
& p_{\bar{d}}^{\mu}=\frac{m_{W}}{2}\left(1, \sin \bar{\theta}^{* *} \cos \bar{\phi}^{* *}, \sin \bar{\theta}^{* *} \sin \bar{\phi}^{* *}, \cos \bar{\theta}^{* *}\right) \\
& p_{u}^{\mu}=\frac{m_{W}}{2}\left(1,-\sin \bar{\theta}^{* *} \cos \bar{\phi}^{* *},-\sin \bar{\theta}^{* *} \sin \bar{\phi}^{* *},-\cos \bar{\theta}^{* *}\right) .
\end{aligned}
$$

Likewise, the $d$ and $\bar{u}$ four momenta are parametrized in the $W^{-} \rightarrow d \bar{u}$ rest frame as

$$
\begin{aligned}
p_{d}^{\mu} & =\frac{m_{W}}{2}\left(1, \sin \theta^{* *} \cos \phi^{* *}, \sin \theta^{* *} \sin \phi^{* *}, \cos \theta^{* *}\right), \\
p_{\bar{u}}^{\mu} & =\frac{m_{W}}{2}\left(1,-\sin \theta^{* *} \cos \phi^{* *},-\sin \theta^{* *} \sin \phi^{* *},-\cos \theta^{* *}\right) .
\end{aligned}
$$

In the $t$ or $\bar{t}$ rest frame, the $\bar{d}$ or $d$ four momenta are obtained from (B.5a) or (B.6a), respectively, by a boost and can be expressed as

$$
\begin{aligned}
p_{\bar{d}}^{\mu} & =\frac{m_{t}}{2}\left(\bar{x}, \bar{x}_{1}, \bar{x}_{2}, \bar{x}_{3}\right), \\
p_{d}^{\mu} & =\frac{m_{t}}{2}\left(x, x_{1}, x_{2}, x_{3}\right) .
\end{aligned}
$$

\footnotetext{
${ }^{5}$ Note that the $\bar{t} \rightarrow \bar{b} \ell^{-} \bar{\nu}$ decay density matrix distributions given in eq. (A30) of ref. [37] differs from (B.1b), because the reference frame in ref. [37] has been chosen to have common $z$ - and $x$-axis for both $t \rightarrow b \ell^{+} \nu$ and $\bar{t} \rightarrow \bar{b} \ell^{-} \bar{\nu}$ decays in the $t \bar{t}$ rest frame in order to study $t$ and $\bar{t}$ decay angular correlations effectively in the process $e^{+} e^{-} \rightarrow h t \bar{t}$.
} 
In ref. [37], it has been assumed that with the probability $P_{\bar{d} u} \geq 0.5$, the $\bar{d}$-quark is correctly identified and with the probability $1-P_{\bar{d} u} \leq 0.5$, the $u$-quark is mistaken as the $\bar{d}$ quark. The $t \rightarrow b \bar{d} u$ decay density matrix distribution is then expressed as

$$
d \rho(t \rightarrow b \bar{d} u)=\frac{6 B(t \rightarrow b \bar{d} u)}{\left(1-\frac{m_{W}^{2}}{m_{t}^{2}}\right)\left(1+2 \frac{m_{W}^{2}}{m_{t}^{2}}\right)}\left[\frac{1+P_{\bar{d} u}}{2} \hat{\rho}_{\bar{d}}+\frac{1-P_{\bar{d} u}}{2} \hat{\rho}_{\bar{d}}^{\prime}\right] \frac{d \cos \theta_{b}^{*} d \phi_{b}^{*}}{4 \pi} \frac{d \cos \bar{\theta}^{* *} d \bar{\phi}^{* *}}{4 \pi}
$$

where

$$
\hat{\rho}_{\bar{d}}=(1-\bar{x})\left(\begin{array}{cc}
\bar{x}+\bar{x}_{3} & \bar{x}_{1}+i \bar{x}_{2} \\
\bar{x}_{1}-i \bar{x}_{2} & \bar{x}-\bar{x}_{3}
\end{array}\right),
$$

and $\hat{\rho}_{\bar{d}}^{\prime}$ is obtained from (B.9) by replacing the $\bar{d}$ and $u$ four momentum (B.5) in the $W^{+}$rest frame. This simple density matrix distribution reduces to the charged lepton distribution (B.1a) in the $P_{\bar{d} u}=1$ limit. The decay density distribution for $\bar{t} \rightarrow \bar{b} \ell \bar{\nu}$ is obtained similarly as

$$
d \rho(\bar{t} \rightarrow \bar{b} \ell \bar{\nu})=\frac{6 B(\bar{t} \rightarrow \bar{b} d \bar{u})}{\left(1-\frac{m_{W}^{2}}{m_{t}^{2}}\right)\left(1+2 \frac{m_{W}^{2}}{m_{t}^{2}}\right)}\left[\frac{1+P_{d \bar{u}}}{2} \hat{\rho}_{d}+\frac{1-P_{d \bar{u}}}{2} \hat{\rho}_{d}^{\prime}\right] \frac{d \cos \theta_{\bar{b}}^{*} d \phi_{\bar{b}}^{*}}{4 \pi} \frac{d \cos \theta^{* *} d \phi^{* *}}{4 \pi}
$$

where the density matrix

$$
\hat{\rho}_{d}=(1-x)\left(\begin{array}{cc}
x-x_{3} & x_{1}-i x_{2} \\
x_{1}+i x_{2} & x+x_{3}
\end{array}\right)
$$

is obtained from the $d$-quark momentum (B.7b) in the $t$-rest frame, and $\hat{\rho}_{d}^{\prime}$ is obtained by exchanging the $d$ and $\bar{u}$ four momenta (B.6) in the same event.

The decay angular distribution of arbitrary polarized $t$ and $\bar{t}$ are then obtained simply by taking the 'trace'

$$
\begin{aligned}
& d \Gamma(t(\vec{P}) \rightarrow b \bar{d} u)=\sum_{\sigma} \sum_{\sigma^{\prime}} \rho_{\sigma \sigma^{\prime}}^{t}(\vec{P}) d \rho(t \rightarrow b \bar{d} u)_{\sigma \sigma^{\prime}} \\
& d \Gamma(\bar{t}(\vec{P}) \rightarrow \bar{b} d \bar{u})=\sum_{\sigma} \sum_{\sigma^{\prime}} \rho_{\sigma \sigma^{\prime}}^{\bar{t}}(\vec{P}) d \rho(\bar{t} \rightarrow \bar{b} d \bar{u})_{\sigma \sigma^{\prime}} .
\end{aligned}
$$

Note that the decay distributions for $t \rightarrow b \bar{s} c$ and $\bar{t} \rightarrow \bar{b} s \bar{c}$ are the same as (B.12a) and (B.12b), respectively, where instead of $\bar{d}$ and $d$ momenta we have $\bar{s}$ and $s$ momenta, while the identification probability $P_{\bar{s} c}=P_{s \bar{c}}$ may be significantly larger than 0.5 , the most pessimistic value which was assumed in ref. [37].

Finally, we find it encouraging that the $t$ and $\bar{t}$ decay angular asymmetries have the same sign when

$$
\vec{P}(\vec{t}) \simeq-\vec{P}(t)
$$

as suggested from approximate CP $\tilde{T}$ invariance in section 6 and from figures 13 and 15 in section 5. This tells that the polarization asymmetry can be measured even if we cannot distinguish $t$ from $\bar{t}$, which may often be the case for hadronic decays. 


\section{Phase space}

The three-body Lorentz invariant phase space for the $t h j$ (or $\bar{t} h j$ ) final state in eq. (3.6) can be parametrized as

$$
d \Phi_{3}(t h j)=d \Phi_{2}(j+t h) \frac{d \mathrm{~W}^{2}}{2 \pi} d \Phi_{2}(t+h),
$$

as a convolution of the two-body phase space integrated over the invariant mass $\mathrm{W}$ of the $t+h$ system

$$
m_{t}+m_{h}<\mathrm{W}<\sqrt{\hat{s}} .
$$

The $j+t h$ phase space

$$
d \Phi_{2}(j+t h)=\frac{1}{8 \pi} x \frac{d \cos \hat{\theta}}{2}
$$

is parametrized in the $u b$ or $t h j$ rest frame, where the four momenta are parametrized as

$$
\begin{aligned}
p_{u} & =\frac{\sqrt{\hat{s}}}{2}(1,0,0,1), \\
p_{b} & =\frac{\sqrt{\hat{s}}}{2}(1,0,0,-1), \\
p_{d} & =\frac{\sqrt{\hat{s}}}{2} x(1, \sin \hat{\theta}, 0, \cos \hat{\theta}), \\
q & =p_{u}-p_{d}=\frac{\sqrt{\hat{s}}}{2}(1-x,-x \sin \hat{\theta}, 0,1-x \cos \hat{\theta}),
\end{aligned}
$$

with $x=1-\mathrm{W}^{2} / \hat{s}$, and

$$
\mathrm{Q}^{2}=-q^{2}=\hat{s} x \frac{1-\cos \hat{\theta}}{2} .
$$

The jet rapidity in the lab frame is hence

$$
\eta_{j}=\frac{1}{2} \ln \frac{1+\cos \hat{\theta}}{1-\cos \hat{\theta}}+Y(t h j) .
$$

The forward peak in the $\eta_{j}$ distribution in figure 5 is due to the square of the common $t$-channel $W$ propagator in the amplitude,

$$
\left|D_{W}(q)\right|^{2}=\frac{1}{\left(\mathbf{Q}^{2}+m_{W}^{2}\right)^{2}}=\frac{1}{\left(\hat{s} x(1-\cos \hat{\theta}) / 2+m_{W}^{2}\right)^{2}}
$$

which grows towards $\cos \hat{\theta} \sim 1$, subject to the jet $p_{T}$ cut

$$
p_{T}^{d}=\frac{\sqrt{\hat{s}}}{2} x \sin \hat{\theta}>30 \mathrm{GeV}
$$

The $t+h$ phase space in the $t h$ rest frame is

$$
d \Phi_{2}(t+h)=\frac{1}{8 \pi} \bar{\beta} \frac{d \cos \theta^{*}}{2} \frac{d \phi}{2 \pi},
$$


where the participating four-momenta for the subprocess $W^{+} b \rightarrow t h$ are parametrized as

$$
\begin{aligned}
q & =\frac{\mathrm{W}}{2}\left(1-\frac{\mathrm{Q}^{2}}{\mathrm{~W}^{2}}, 0,0,1+\frac{\mathrm{Q}^{2}}{\mathrm{~W}^{2}}\right), \\
p_{b} & =\frac{\mathrm{W}}{2}\left(1+\frac{\mathrm{Q}^{2}}{\mathrm{~W}^{2}}, 0,0,-1-\frac{\mathrm{Q}^{2}}{\mathrm{~W}^{2}}\right), \\
p_{t} & =\frac{\mathrm{W}}{2}\left(1+\frac{m_{t}^{2}-m_{h}^{2}}{\mathrm{~W}^{2}}, \bar{\beta} \sin \theta^{*} \cos \phi, \bar{\beta} \sin \theta^{*} \sin \phi, \bar{\beta} \cos \theta^{*}\right), \\
p_{h} & =\frac{\mathrm{W}}{2}\left(1+\frac{m_{h}^{2}-m_{t}^{2}}{\mathrm{~W}^{2}},-\bar{\beta} \sin \theta^{*} \cos \phi,-\bar{\beta} \sin \theta^{*} \sin \phi,-\bar{\beta} \cos \theta^{*}\right) .
\end{aligned}
$$

In this frame where the virtual $W$ momentum $\vec{q}$ is chosen along the positive $z$-axis, eq. (C.10a), the up and down quark have the same transverse momentum. Their four momenta are obtained from eqs. (C.4a), (C.4c) by a rotation about the $y$-axis, and can be expressed in the Breit frame as

$$
\begin{aligned}
p_{u} & =\tilde{\omega}(1, \sin \tilde{\theta}, 0, \cos \tilde{\theta}), \\
p_{d} & =\tilde{\omega}(1, \sin \tilde{\theta}, 0,-\cos \tilde{\theta}),
\end{aligned}
$$

where $\tilde{\omega}$ and $\cos \tilde{\theta}$ are given by eq. (2.14). In this frame, the azimuthal angle $\phi$ of the top quark in eq. (C.10) is measured from the $x-z$ plane defined by the $u \rightarrow d W^{+}$emission, given by eq. (C.11). We evaluate the helicity amplitudes in the frame where the azimuthal angle $\phi$ is given to the $u$ and $d$ quark, as in eq. (2.13), while the top quark is produced in the $x$ - $z$ plane, as in eq. (2.11). It is in this particular Lorentz frame, obtained by a rotation of $-\phi$ about the $z$-axis depicted in figure 3, that the helicity amplitudes for the process $u b \rightarrow d t h$ can be expressed as a combination of the $u \rightarrow d W^{ \pm}$emission amplitudes with the $e^{ \pm i \phi}$ phase factors, eq. (2.15), and the $W^{+} b \rightarrow t h$ production amplitudes of eq. (2.22).

\section{Acknowledgments}

We are grateful to Tie-Jiun Hou, Junichi Kanzaki and Kentarou Mawatari for helpful discussions. YZ would like to thank Haider Alhazmi, Sally Dawson, Samuel Lane, Ian Lewis and Kun Liu for valuable suggestions. KH and YZ thank Tao Han and the members of PITT-PACC and Fermilab Theory Division for their warm hospitality, where part of the present work was carried out. This work has been supported in part by the U.S. Department of Energy under contract number DE-SC-0017647. YZ is supported by the U.S. Department of Energy under grant No. DE-SC0019474. 
Open Access. This article is distributed under the terms of the Creative Commons Attribution License (CC-BY 4.0), which permits any use, distribution and reproduction in any medium, provided the original author(s) and source are credited.

\section{References}

[1] CMS collaboration, Observation of t $\bar{t} H$ production, Phys. Rev. Lett. 120 (2018) 231801 [arXiv: 1804.02610] [INSPIRE].

[2] ATLAS collaboration, Observation of Higgs boson production in association with a top quark pair at the LHC with the ATLAS detector, Phys. Lett. B 784 (2018) 173 [arXiv: 1806.00425] [INSPIRE].

[3] W. Stirling and D.J. Summers, Production of an intermediate mass Higgs boson in association with a single top quark at LHC and SSC, Phys. Lett. B 283 (1992) 411 [INSPIRE].

[4] G. Bordes and B. van Eijk, On the associate production of a neutral intermediate mass Higgs boson with a single top quark at the LHC and SSC, Phys. Lett. B 299 (1993) 315 [INSPIRE].

[5] ATLAS collaboration, Measurements of gluon-gluon fusion and vector-boson fusion Higgs

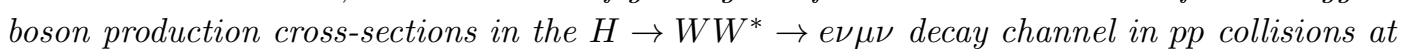
$\sqrt{s}=13 \mathrm{TeV}$ with the ATLAS detector, Phys. Lett. B 789 (2019) 508 [arXiv:1808.09054] [INSPIRE].

[6] ATLAS collaboration, Measurement of the production cross section for a Higgs boson in association with a vector boson in the $H \rightarrow W W^{*} \rightarrow \ell \nu \ell \nu$ channel in pp collisions at $\sqrt{s}=13$ TeV with the ATLAS detector, Phys. Lett. B 798 (2019) 134949 [arXiv: 1903.10052] [INSPIRE].

[7] CMS collaboration, Measurements of properties of the Higgs boson decaying to a $W$ boson pair in pp collisions at $\sqrt{s}=13 \mathrm{TeV}$, Phys. Lett. B 791 (2019) 96 [arXiv:1806.05246] [INSPIRE].

[8] M. Cepeda et al., Report from Working Group 2: Higgs Physics at the HL-LHC and HE-LHC, CERN Yellow Rep. Monogr. 7 (2019) 221 [arXiv:1902.00134] [INSPIRE].

[9] J. Alwall et al., The automated computation of tree-level and next-to-leading order differential cross sections, and their matching to parton shower simulations, JHEP 07 (2014) 079 [arXiv: 1405.0301] [INSPIRE].

[10] A. Alloul, N.D. Christensen, C. Degrande, C. Duhr and B. Fuks, FeynRules $2.0-A$ complete toolbox for tree-level phenomenology, Comput. Phys. Commun. 185 (2014) 2250 [arXiv: 1310.1921] [INSPIRE].

[11] F. Demartin, F. Maltoni, K. Mawatari and M. Zaro, Higgs production in association with a single top quark at the LHC, Eur. Phys. J. C 75 (2015) 267 [arXiv: 1504.00611] [InSPIRE].

[12] W. Beenakker, S. Dittmaier, M. Krämer, B. Plumper, M. Spira and P.M. Zerwas, NLO QCD

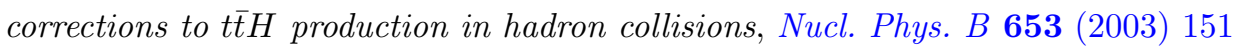
[hep-ph/0211352] [INSPIRE].

[13] Particle Data Group collaboration, Review of Particle Physics, Phys. Rev. D 98 (2018) 030001 [INSPIRE]. 
[14] CMS collaboration, Search for the associated production of a Higgs boson with a single top quark in proton-proton collisions at $\sqrt{s}=8 \mathrm{TeV}$, JHEP 06 (2016) 177 [arXiv:1509.08159] [INSPIRE].

[15] CMS collaboration, Search for production of a Higgs boson and a single top quark in multilepton final states in proton collisions at $\sqrt{s}=13 \mathrm{TeV}$, CMS-PAS-HIG-17-005 (2017).

[16] CMS collaboration, Search for resonant pair production of Higgs bosons decaying to bottom quark-antiquark pairs in proton-proton collisions at 13 TeV, CMS-PAS-HIG-17-009 (2017).

[17] CMS collaboration, Search for the $t H(H \rightarrow b \bar{b})$ process in pp collisions at $\sqrt{s}=13 \mathrm{TeV}$ and study of Higgs boson couplings, CMS-PAS-HIG-17-016 (2018).

[18] CMS collaboration, Search for associated production of a Higgs boson and a single top quark in proton-proton collisions at $\sqrt{s}=13$ TeV, Phys. Rev. D 99 (2019) 092005 [arXiv: 1811.09696] [INSPIRE].

[19] F. Maltoni, K. Paul, T. Stelzer and S. Willenbrock, Associated production of Higgs and single top at hadron colliders, Phys. Rev. D 64 (2001) 094023 [hep-ph/0106293] [INSPIRE].

[20] V. Barger, M. McCaskey and G. Shaughnessy, Single top and Higgs associated production at the LHC, Phys. Rev. D 81 (2010) 034020 [arXiv:0911.1556] [InSPIRE].

[21] J. Chang, K. Cheung, J.S. Lee and C.-T. Lu, Probing the Top-Yukawa Coupling in Associated Higgs production with a Single Top Quark, JHEP 05 (2014) 062 [arXiv:1403.2053] [INSPIRE].

[22] S. Biswas, E. Gabrielli and B. Mele, Single top and Higgs associated production as a probe of the Htt coupling sign at the LHC, JHEP 01 (2013) 088 [arXiv:1211.0499] [INSPIRE].

[23] J. Yue, Enhanced thj signal at the LHC with $h \rightarrow \gamma \gamma$ decay and $\mathcal{C P}$-violating top-Higgs coupling, Phys. Lett. B 744 (2015) 131 [arXiv:1410.2701] [INSPIRE].

[24] A.V. Gritsan, R. Röntsch, M. Schulze and M. Xiao, Constraining anomalous Higgs boson couplings to the heavy flavor fermions using matrix element techniques, Phys. Rev. D 94 (2016) 055023 [arXiv: 1606.03107] [INSPIRE].

[25] M. Farina, C. Grojean, F. Maltoni, E. Salvioni and A. Thamm, Lifting degeneracies in Higgs couplings using single top production in association with a Higgs boson, JHEP 05 (2013) 022 [arXiv: 1211.3736] [INSPIRE].

[26] P. Agrawal, S. Mitra and A. Shivaji, Effect of Anomalous Couplings on the Associated Production of a Single Top Quark and a Higgs Boson at the LHC, JHEP 12 (2013) 077 [arXiv: 1211.4362] [INSPIRE].

[27] A. Kobakhidze, L. Wu and J. Yue, Anomalous Top-Higgs Couplings and Top Polarisation in Single Top and Higgs Associated Production at the LHC, JHEP 10 (2014) 100 [arXiv:1406.1961] [INSPIRE].

[28] D. Atwood, S. Bar-Shalom, G. Eilam and A. Soni, CP violation in top physics, Phys. Rept. 347 (2001) 1 [hep-ph/0006032] [INSPIRE].

[29] S.D. Rindani, P. Sharma and A. Shivaji, Unraveling the CP phase of top-Higgs coupling in associated production at the LHC, Phys. Lett. B 761 (2016) 25 [arXiv:1605.03806] [INSPIRE].

[30] M. Kraus, T. Martini, S. Peitzsch and P. Uwer, Exploring BSM Higgs couplings in single top-quark production, arXiv: 1908.09100 [INSPIRE]. 
[31] A. Djouadi, The Anatomy of electro-weak symmetry breaking. II. The Higgs bosons in the minimal supersymmetric model, Phys. Rept. 459 (2008) 1 [hep-ph/0503173] [INSPIRE].

[32] V. Barger, K. Hagiwara and Y.-J. Zheng, Probing the Higgs Yukawa coupling to the top quark at the LHC via single top+Higgs production, Phys. Rev. D 99 (2019) 031701 [arXiv: 1807.00281] [INSPIRE].

[33] D.A. Faroughy, J.F. Kamenik, N. Košnik and A. Smolkovič, Probing the CP nature of the top quark Yukawa at hadron colliders, JHEP 02 (2020) 085 [arXiv: 1909.00007] [INSPIRE].

[34] K. Hagiwara, Q. Li and K. Mawatari, Jet angular correlation in vector-boson fusion processes at hadron colliders, JHEP 07 (2009) 101 [arXiv: 0905.4314] [INSPIRE].

[35] K. Hagiwara and D. Zeppenfeld, Helicity Amplitudes for Heavy Lepton Production in $e^{+} e^{-}$ Annihilation, Nucl. Phys. B 274 (1986) 1 [InSPIRE].

[36] K. Hagiwara, H. Murayama and I. Watanabe, Search for the Yukawa interaction in the process $e^{+} e^{-} \rightarrow t \bar{t} Z$ at TeV linear colliders, Nucl. Phys. B 367 (1991) 257 [inSPIRE].

[37] K. Hagiwara, H. Yokoya and Y.-J. Zheng, Probing the CP properties of top Yukawa coupling at an $e^{+} e^{-}$collider, JHEP 02 (2018) 180 [arXiv: 1712.09953] [INSPIRE].

[38] S. Dulat et al., New parton distribution functions from a global analysis of quantum chromodynamics, Phys. Rev. D 93 (2016) 033006 [arXiv: 1506.07443] [INSPIRE].

[39] D. Atwood, A. Aeppli and A. Soni, Extracting anomalous gluon-top effective couplings at the supercolliders, Phys. Rev. Lett. 69 (1992) 2754 [INSPIRE].

[40] A. De Rujula, R. Petronzio and B.E. Lautrup, On the Challenge of Measuring the Color Charge of Gluons, Nucl. Phys. B 146 (1978) 50 [InSPIRE].

[41] K. Hagiwara, K.-i. Hikasa and N. Kai, Time Reversal Odd Asymmetry in Semiinclusive Leptoproduction in Quantum Chromodynamics, Phys. Rev. D 27 (1983) 84 [INSPIRE].

[42] K. Hagiwara, R.D. Peccei, D. Zeppenfeld and K. Hikasa, Probing the Weak Boson Sector in $e^{+} e^{-} \rightarrow W^{+} W^{-}$, Nucl. Phys. B 282 (1987) 253 [InSPIRE].

[43] V. Barger, K. Hagiwara and Y.J. Zheng, T-odd asymmetries from radiative corrections in the $p p \rightarrow$ thj process, in preparation. 